\title{
Optimization Models for Three On-Chip Network Problems
}

\author{
NILAY VAISH, MICHAEL C. FERRIS, and DAVID A. WOOD, University of Wisconsin-Madison
}

\begin{abstract}
We model three on-chip network design problems-memory controller placement, resource allocation in heterogeneous on-chip networks, and their combination-as mathematical optimization problems. We model the first two problems as mixed integer linear programs. We model the third problem as a mixed integer nonlinear program, which we then linearize exactly. Sophisticated optimization algorithms enable solutions to be obtained much more efficiently. Detailed simulations using synthetic traffic and benchmark applications validate that our designs provide better performance than solutions proposed previously. Our work provides further evidence toward suitability of optimization models in searching/pruning architectural design space.
\end{abstract}

CCS Concepts: • Computer systems organization $\rightarrow$ Interconnection architectures; Multicore architectures

Additional Key Words and Phrases: On-chip network, optimization models

ACM Reference Format:

Nilay Vaish, Michael C. Ferris, and David A. Wood. 2016. Optimization models for three on-chip network problems. ACM Trans. Archit. Code Optim. 13, 3, Article 26 (September 2016), 27 pages.

DOI: http://dx.doi.org/10.1145/2943781

\section{INTRODUCTION}

As Moore's law continues to deliver exponential increases in the number of transistors, chip multiprocessors have begun to move from multicores to many-cores. As a result, designers are facing a combinatorial explosion in the design space, with an enormous number of ways (quantified in later sections) to allocate a chip's limited resources among cores, caches, on-chip interconnect, and memory controllers. Different designs have different computation and communication performance. Therefore, we need to search the design space for the best possible designs. But even highly constrained resource allocation problems, such as where to place memory controllers in the network [Abts et al. 2009], result in far more configurations than can be evaluated using traditional simulation-based techniques. Hence, we need models that can help in exploring the design space efficiently.

In this work, ${ }^{1}$ we present mathematical optimization-based models for three problems related to on-chip networks for tiled processors like one shown in Figure 1. We

\footnotetext{
${ }^{1} \mathrm{~A}$ portion of this work appears in Chapter 6 of Nowatzki et al. [2013]. The work presented in Sections 3 and 4 does not appear in Nowatzki et al. The work presented in Section 5 has more detailed analysis and

This material is based on work partially supported by the U.S. Department of Energy, Office of Science, Office of Advanced Scientific Computing Research, Applied Mathematics program under contract DE-AC0206CH11357 through the Project "Multifaceted Mathematics for Complex Energy Systems"; AFOSR grant FA9550-15-1-0212; and NSF grants IIS-1227530, CCF-1218323, CNS-1302260, CCF-1438992, and CCF1533885. Professor Wood has a significant financial interest in AMD.

Authors' address: N. Vaish, M. C. Ferris, and D. A. Wood, Department of Computer Sciences, University of Wisconsin-Madison, 1210 W. Dayton Street, Madison, WI 53706; emails: \{nilay, ferris, david\}@cs.wisc.edu. Permission to make digital or hard copies of part or all of this work for personal or classroom use is granted without fee provided that copies are not made or distributed for profit or commercial advantage and that copies show this notice on the first page or initial screen of a display along with the full citation. Copyrights for components of this work owned by others than ACM must be honored. Abstracting with credit is permitted. To copy otherwise, to republish, to post on servers, to redistribute to lists, or to use any component of this work in other works requires prior specific permission and/or a fee. Permissions may be requested from Publications Dept., ACM, Inc., 2 Penn Plaza, Suite 701, New York, NY 10121-0701 USA, fax +1 (212) 869-0481, or permissions@acm.org.
}

(c) 2016 ACM 1544-3566/2016/09-ART26 $\$ 15.00$

DOI: http://dx.doi.org/10.1145/2943781 


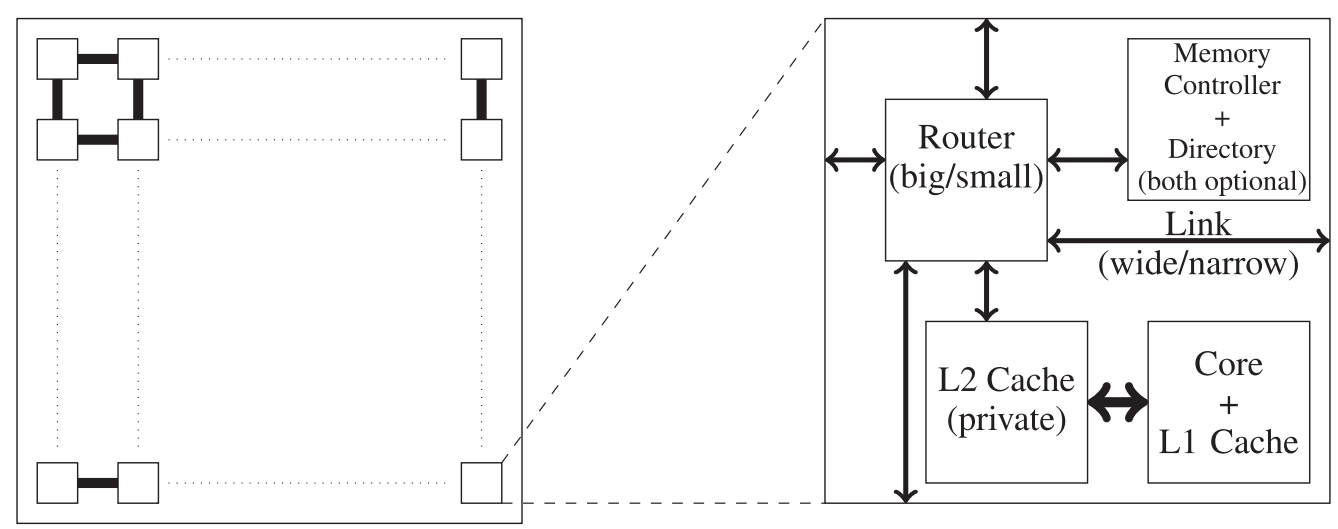

Fig. 1. Processor layout (not to scale). On the left is an $n \times n$-tiled processor. The tiles are connected using a mesh/torus network. The figure on the right shows a single tile. The memory controller is optional. Routers for different tiles may have different amounts of resources. The links in between tiles can be wide or narrow.

assume that the processor has a homogeneous set of cores, each with two levels of private caches. These caches are kept coherent using a directory-based MOESI coherence protocol. The first two problems we model are memory controller placement [Abts et al. 2009] (Section 3) and resource allocation in heterogeneous on-chip networks [Mishra et al. 2011] (Section 4). The combination of these two problems is a much more challenging nonconvex optimization problem but explores the trade-offs between shared resources in the design (Section 5). We present our computational experience with the nonconvex model and an equivalent linearized model, which can be solved much more efficiently.

Simulation-based experiments provide evidence that our solution for the combined problem provides $5.4 \%$ and $22 \%$ better performance on multiprogrammed workloads composed of applications from SPEC CPU2006 [Henning 2006] and NAS Parallel Benchmarks (NPB) [Bailey et al. 1991], respectively. Although mathematical optimization has been used previously in different facets of computer architecture, including on-chip network design [Srinivasan et al. 2004; Kinsy et al. 2009; Marculescu and Bogdan 2009], compilers [Fu and Wilken 2002; Abdel-Gawad and Thottethodi 2011], and design space exploration [Azizi et al. 2010], we believe that we are the first to use it for these three problems.

\section{OVERVIEW OF MATHEMATICAL OPTIMIZATION}

Mathematical optimization deals with the problem of making the best possible choice from a large set of feasible alternatives. Choices are expressed as values taken by a collection of variables. Feasible choices are specified by constraints on the values of these variables. A designated function that evaluates a particular criterion quantifies the best choice. Mathematical optimization helps us make the best possible choice efficiently using the power of calculus, logic, and mathematics.

Formally, an optimization problem has the following form [Boyd and Vandenberghe 2004]:

$$
\begin{array}{rc}
\text { minimize } & f_{0}(x) \\
\text { subject to } & f_{i}(x) \leq 0, \quad i=1, \ldots, m
\end{array}
$$

evaluation of the model and of the designs obtained as solutions to the model. Figures 17 and 18 have been reused from Nowatzki et al. with permission from Morgan \& Claypool Publishers. 
Here, $x=\left(x_{1}, \ldots, x_{n}\right)$ is the vector of optimization variables, the function $f_{0}: \mathbb{R}^{n} \rightarrow \mathbb{R}$ is the objective function, and the functions $f_{i}: \mathbb{R}^{n} \rightarrow \mathbb{R}$ are the constraint functions. A feasible vector $x^{*}$ is called optimal if it has the smallest objective function value among all vectors $z \in \mathbb{R}^{n}$ that satisfy the constraints-that is, $f_{0}\left(x^{*}\right) \leq f_{0}(z)$ for any $z$ for which $f_{i}(z) \leq 0, i=1, \ldots, m$.

\subsection{Types of Optimization Problems}

Optimization problems can be divided into several different classes depending on the particular forms of the objective and the constraint functions. In our work, we make use of two different classes:

-Mixed integer linear program (MILP): In an MILP, the objective and the constraint functions are linear and one or more variables in the program take on integer-only values.

- Mixed integer nonlinear program (MINLP): This class includes problems that have at least one function (objective or constraint) that is not linear. Additionally, one or more variables take integer-only values.

Theoretically, problems in these classes are known to be NP-hard [Papadimitriou 1981]. Over the years, researchers have developed algorithms and tools that in many instances can solve these problems efficiently. We briefly describe the tools used in our work. The General Algebraic Modeling System (GAMS) [GAMS Development Corporation 2015] is a language for representing optimization models. A particular data instance of the model is compiled into an efficient scalar representation, which is passed on to a solver. A solver is a tool that implements algorithms for solving optimization models. For example, Gurobi [Gurobi Optimization, Inc. 2015] is a solver for mixed integer linear and convex quadratic optimization problems, and Baron [Tawarmalani and Sahinidis 2005 ] is a solver for optimization problems defined with general nonlinear functions $f_{i}$. Although a solver may not always be successful in finding an optimal solution for the model due to NP-hardness, the preceding solvers give solution guarantees relating the values found to the best possible ones.

\subsection{Convexity}

Certain mathematical concepts facilitate a more efficient solution of these problems. We use notions related to convexity throughout this article; interested readers may go through any standard text on mathematical optimization for a detailed discussion (e.g., Boyd and Vandenberghe [2004]). We use the following core concepts:

-Convex set: A set $C \subseteq \mathbb{R}^{n}$ is said to be convex if for any $x_{1}, x_{2} \in C$ and any $\theta$ with $0 \leq \theta \leq 1$, we have $\theta x_{1}+(1-\theta) x_{2} \in C$.

Convex function: A function $f: D \rightarrow \mathbb{R}$ is called convex if its domain $D \subset \mathbb{R}^{n}$ is a convex set and for all $x, y \in D$, and any $\theta$ with $0 \leq \theta \leq 1$, we have $f(\theta x+(1-\theta) y) \leq$ $\theta f(x)+(1-\theta) f(y)$.

-An optimization problem is called convex if it is of the form

$$
\begin{array}{cc}
\text { minimize } & f_{0}(x) \\
\text { subject to } & f_{i}(x) \leq 0, \quad i=1, \ldots, m \\
& a_{i}^{T} x=b_{i}, \quad i=1, \ldots, p,
\end{array}
$$

where $f_{0}, \ldots, f_{m}$ are convex functions, $a_{i}$ are $n$-dimensional real vector constants, and $b_{i}$ are scalar constants.

A point $x^{*} \in \mathbb{R}^{n}$ is locally optimal if it is feasible and its objective value is no worse than the objective value at all neighboring feasible points. It is globally optimal if the 


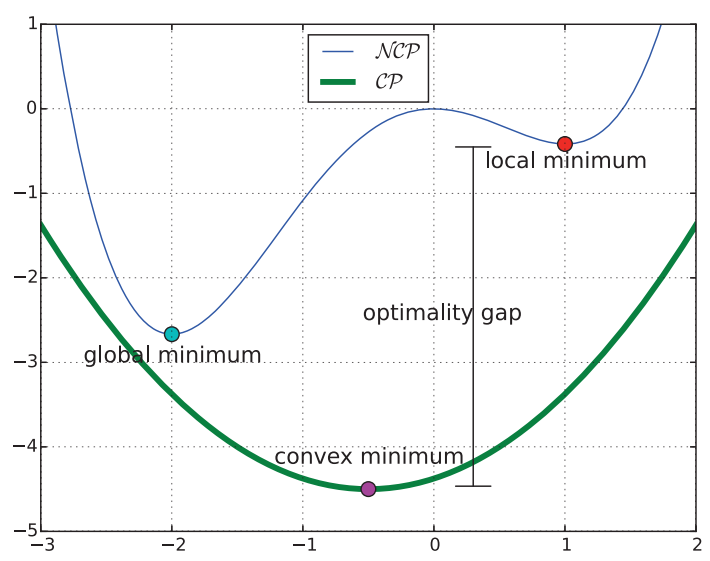

Fig. 2. Example of convex underestimation. $\mathcal{N C P}$ is nonconvex function, and $\mathcal{C P}$ is its convex underestimate. Suppose that some algorithm outputs the local minimum (shown in red) for $\mathcal{N} \mathcal{C P}$. The minimum for $\mathcal{C P}$ can be used to compute the maximum possible gap between the unknown global minimum for $\mathcal{N C P}$ and the known local minimum.

neighborhood is the whole space. For a convex optimization problem, any locally optimal solution is globally optimal. Local optimality is easily verified, and thus efficient methods exist for solving convex problems.

Nonconvex optimization problems, on the other hand, do not have efficient solution procedures in general. Methods exist that can provide solutions that are locally optimal, but such a solution might be very far from the global optimum. In particular cases, it is possible to use convex problems to approximate or even exactly solve nonconvex problems. For example, suppose that we need to a solve a nonconvex minimization problem $\mathcal{N C P}$. One may construct a convex problem, $\mathcal{C P}$, such that the objective function value for $\mathcal{C P}$ is at most the objective function value for $\mathcal{N C P}$ at all feasible points (i.e., $\mathcal{C P}$ underestimates $\mathcal{N C P}$ ). Then, the optimal objective function value for $\mathcal{C P}$ gives us a lower bound on the optimal objective function value for $\mathcal{N C P}$. We show this pictorially in Figure 2. For integer linear programs, such bounds can be obtained by removing the integrality constraints. The bound can be used to compare the quality of any given feasible solution with the unknown optimal solution.

\subsection{Optimization for Design Space Exploration}

In our work, we focus on placement and allocation of network resources for many-core designs. The design space is so large that it is infeasible to enumerate/simulate its entirety. But the performance objective and the design constraints can be effectively modeled using integer variables combined with linear/convex functions. Although these optimization models are not as detailed as a simulation model, they are good enough to guide the exploration to regions of good performance. This is confirmed through simulation experiments, where our designs perform better than solutions proposed previously.

\section{PLACEMENT OF MEMORY CONTROLLERS}

Chip multiprocessors (CMPs) need abundant DRAM bandwidth to feed the increasing number of cores. Due to limited pin bandwidth [Abts et al. 2009], there will be more cores compared to the number of memory controllers on a chip. For example, the Oracle Sparc T5 [Feehrer et al. 2013] has four memory controllers for 16 cores. This raises the question of how to place the memory controllers within the on-chip network. Careful 


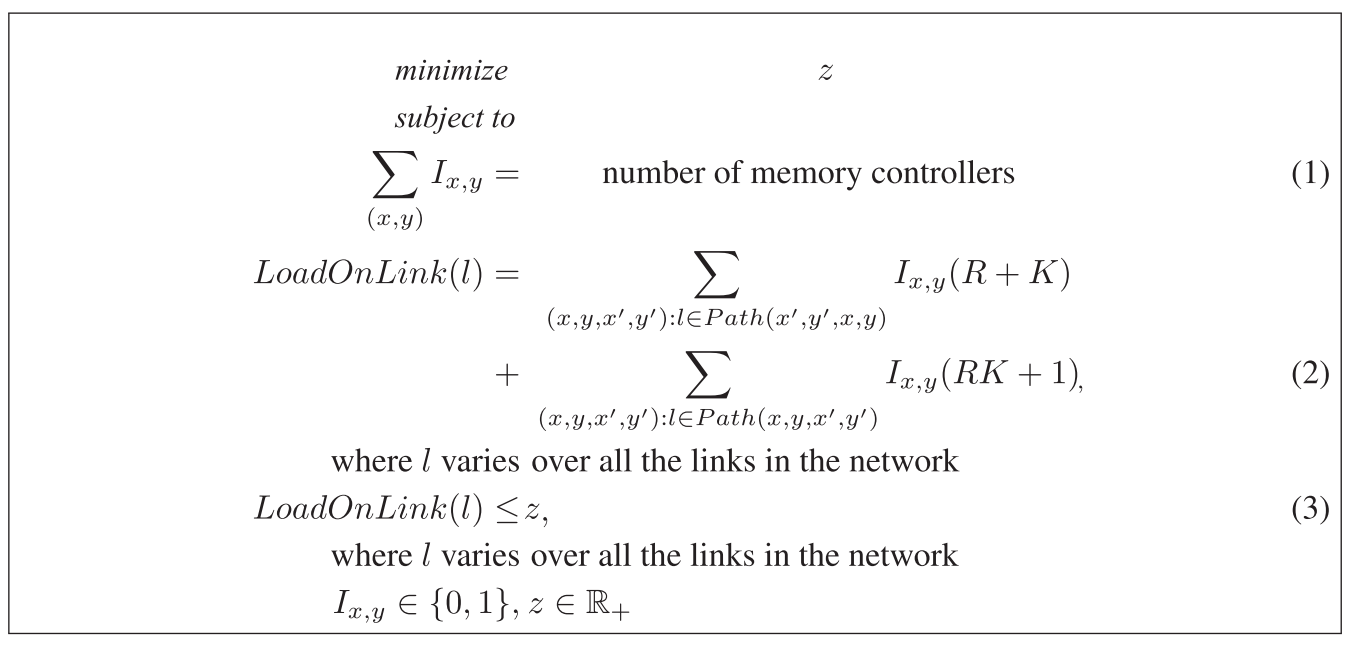

Fig. 3. MILP for placing memory controllers.

placement of the controllers can lead to lower latency and better bandwidth utilization for on-chip communication. This problem was introduced by Abts et al. [2009].

Consider a design problem with $n$ cores and $m$ memory ports. Assuming that the memory ports are co-located with the cores, there are $\left(\begin{array}{l}n \\ m\end{array}\right)$ possible ways of placing the memory controllers. For a 64-core, 16-port design, this number is about $4.9 \times 10^{14}$. It is not possible to explore each and every placement of the memory controllers. Abts et al. [2009] search this design space using a combination of intuition (experience), exhaustive simulation of smaller designs, and genetic algorithm (GA)-based approach to arrive at a reasonably good placement. Instead, we use mathematical optimization for searching the design space by creating an optimization-based model for the problem. We next discuss the assumptions used in the model.

\subsection{Assumptions}

Similar to Abts et al. [2009], we assume that the cores are laid out on a 2D plane and are connected using an on-chip network. The memory ports are co-located with the cores. The on-chip network uses a deterministic routing protocol-that is, all messages from node A to node B always traverse the same path [Enright Jerger and Peh 2009]. Last, since CMPs typically distribute memory addresses across controllers using lower-order address bits [Feehrer et al. 2013], we assume that the traffic is distributed uniformly across all memory controllers.

Since the memory controllers are distributed (possibly) over the entire chip area, the chip would need to be manufactured such that the I/O pins cover the entire area of the chip and not just the periphery, as is the case with flip chip technology [Tong et al. 2013]. The design constraints on these I/O pins are not considered in our model. We also ignore the difference in areas of cores that have memory ports and those without.

\subsection{Notation Used in the Model}

We model the problem as an MILP. Figure 3 shows the formulated model. In the model, each memory controller is denoted as a point $(x, y)$ on the 2D plane. Similarly, a core is referred to with coordinates $\left(x^{\prime}, y^{\prime}\right)$. For each $(x, y), I_{x, y}$ is a binary variable denoting whether a memory controller is placed at $(x, y)$. LoadOnLink $(l)$ denotes the load on the link $l$ due to the communication between the cores and the memory controllers. This 


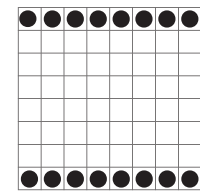

(a) row 0-7

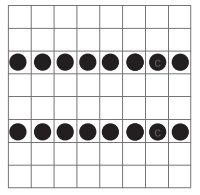

(b) row 2-5

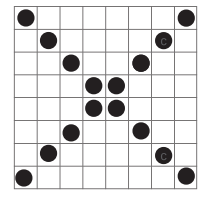

(c) diagonal

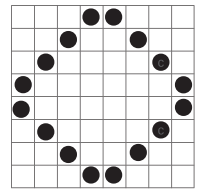

(d) diamond

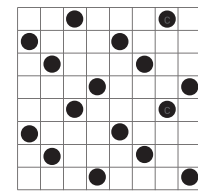

(e) mc-opt

Fig. 4. Different controller placements for an $8 \times 8$ mesh/torus network. Tiles with a black-colored sphere represent a memory port co-located with a core.

load depends on the placement of the controllers. The set Path $\left(x, y, x^{\prime}, y^{\prime}\right)$ contains all links $l$ that are used for going from $(x, y)$ to $\left(x^{\prime}, y^{\prime}\right)$; since the routing protocol is deterministic, Path() is an input to the problem. $z$ bounds the maximum load a link can be assigned. We further assume that the read to write requests have a ratio of $R: 1$ and that a packet with data is $K$-times the size of a packet with no data.

\subsection{Description of the Model}

There are three constraints involved in the model:

-Equation (1) enforces that a specified number of memory controllers $(m)$ are placed on the chip. $I_{x, y}$ can be either 0 or 1 . Thus, when the equation is satisfied, exactly $m$ of the $n I_{x, y}$-variables are set to 1 and the rest are 0 .

-Equation (2) defines the load on each link in the on-chip network. The first term on the right-hand side of the constraint is a sum over all pairs $(x, y)$ and $\left(x^{\prime}, y^{\prime}\right)$ such that a memory controller is placed at $(x, y)$ and the path from $\left(x^{\prime}, y^{\prime}\right)$ to $(x, y)$ uses link $l$. This term represents the request traffic going from the cores to the memory controllers. We assume that the traffic has an $R: 1$ ratio for reads and writes. Each read request requires a single flit, whereas a write request needs $K$ flits. Hence, the total request traffic is proportional to $R+K$. The second term, which represents the response traffic from the memory controllers to the cores, can be interpreted in a similar fashion. The total response traffic is proportional to $R K+1$, where $R K$ is for flits with response data for read requests and 1 is for flits with acknowledgment for write requests.

-Equation (3) bounds the load on any link to be at most as large as $z$.

The objective of the problem is to minimize $z$, the maximum load on any of the linksthat is, the maximum channel load. Thus, an optimal solution for the model would place the memory controllers such that the maximum load placed on any of the links is as low as possible. A lower value for $z$ means less congestion in the network and lower queueing delays. Alternately, we would like to use the available bandwidth efficiently by spreading the load across as many links as possible in a uniform manner. We chose this objective to keep the model linear.

\subsection{Solving the Model}

For solving the model, we assume that the cores are connected using a $k \times k 2 \mathrm{D}$ mesh or torus on-chip network. The number of memory controllers that need to be placed is $2 k$. We also assume that the network uses dimension-ordered routing. Abts et al. [2009] made similar assumptions.

We express the model using GAMS [GAMS Development Corporation 2015] and solve it using Gurobi [Gurobi Optimization, Inc. 2015]. Figure 4 shows some of the possible placements for $k=8$. The diamond placement, shown in Figure 4(d), was reported as the best placement by Abts et al. [2009]. Figure 4(e), herein termed as mc-opt, is the placement obtained as a solution to the model when $R$ and $K$ are both set to 1 . The 


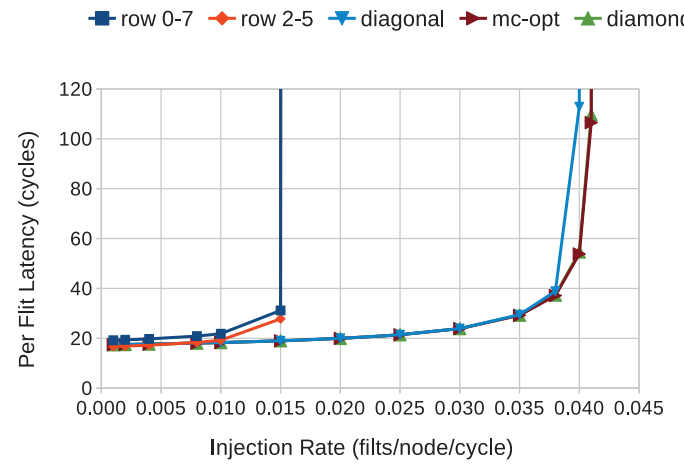

(a) Mesh network

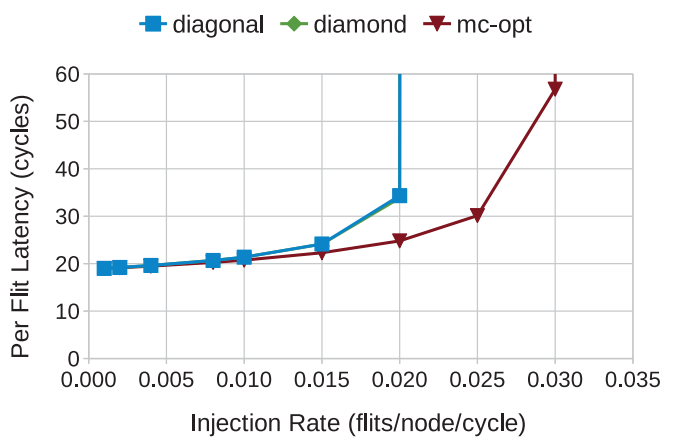

(b) Torus network

Fig. 5. Average latency versus injection rate (uniform random traffic).

same placement is optimal for both mesh and torus networks. Note that many other placements are optimal as well.

\subsection{Evaluation of the Model}

Our model takes a simplified view of an on-chip network. To validate that the simplified view is sufficient for the purpose of pruning the design space, we carry out different simulation-based experiments. All experiments use $8 \times 8$ mesh and torus networks.

3.5.1. Performance with Equiprobable Read and Write Synthetic Traffic. Our first experiment uses a detailed on-chip network simulator, which is part of gem5 [Binkert et al. 2011] and based on GARNET [Agarwal et al. 2009]. During the simulation, all cores inject request packets into the network at a fixed rate. The request addresses are generated in a manner so that the requests are uniformly distributed among the memory controllers. The memory controllers then respond to these requests, thus completing the requestresponse loop. Statistical results are collected after running the simulation for 2 million cycles.

Figure 5 shows the plots of average flit latency versus the rate of request injection. Figure 5(a) is for a mesh network, whereas Figure 5(b) is for a torus network. From the graphs, we conclude that for the mesh network, mc-opt has performance similar to that of diamond and diagonal. For the torus network, mc-opt supports about a $50 \%$ higher request injection rate before saturation compared to diagonal and diamond. It also provides lower average latency. Thus, the design obtained from our optimization-based model works well when the traffic is uniformly distributed.

3.5.2. Performance with SPEC CPU2006 Applications. In our second experiment, we evaluate the designs by simulating applications from the SPEC CPU2006 benchmark suite [Henning 2006] on the gem5 simulator. Such an experiment evaluates the efficacy of the design suggested by the optimization-based model in a close to realistic situation.

We briefly describe our experimental setup. We chose 10 applications from the suite, namely astar, lbm, mcf, milc, omnetpp, libquantum, leslie3d, soplex, sphinx3, and GemsFDTD. We created checkpoints for these applications after skipping the first 100 billion instructions. For each simulation, we dropped two applications and simulated eight copies of each of the eight remaining applications. These applications were mapped to the cores randomly. Each simulation was allowed to run until every core had executed at least 25 million instructions. On average, about 4 billion instructions were 
Table I. Processor and Cache Simulation Parameters

\begin{tabular}{l|l}
\hline Parameter & \multicolumn{1}{|c}{ Value } \\
\hline Processors & 64 out-of-order cores operating at 2GHz \\
\hline L1 I cache & 32KB, 2-way set associative, \\
& 2-cycle latency \\
\hline L1 D cache & 64KB, 2-way set associative, \\
& 2-cycle latency \\
\hline L2 cache (private) & 2MB, 8-way set associative, \\
& 10-cycle latency \\
\hline Main memory & 48GB, 16 DDR-style controllers, \\
& addressed using bits 15:12. \\
\hline
\end{tabular}

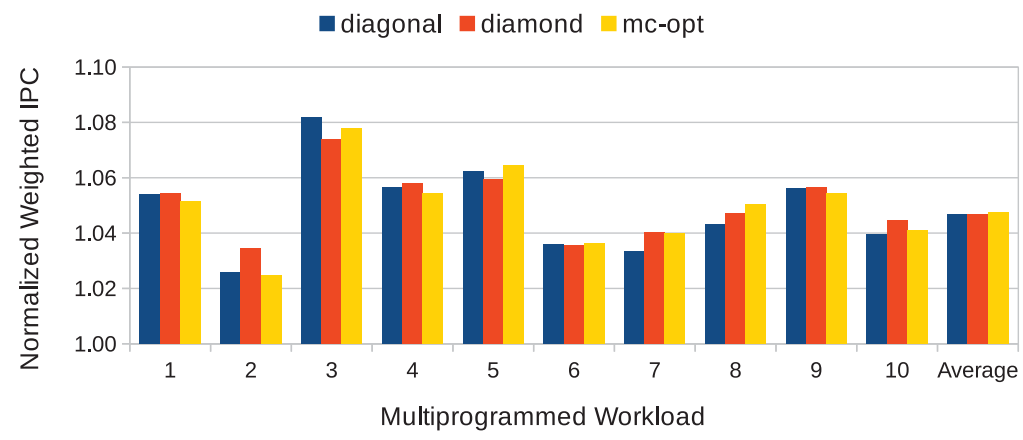

(a) Mesh network

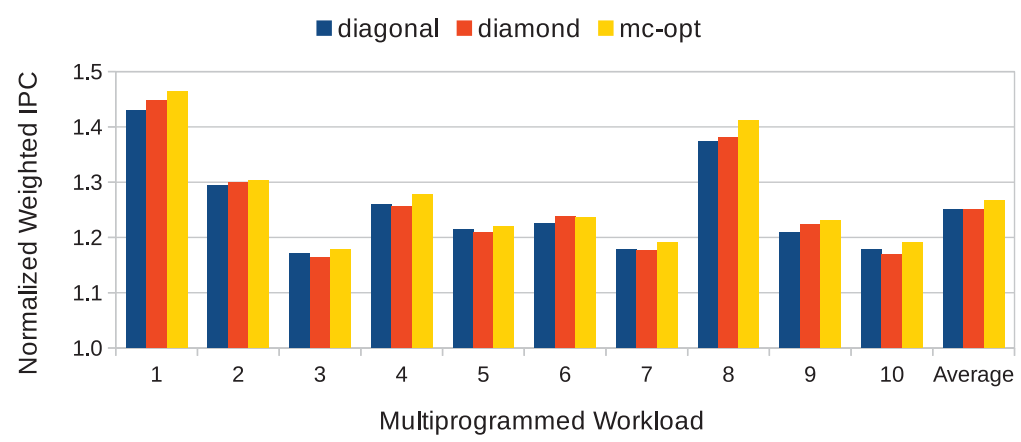

(b) Torus network

Fig. 6. Weighted speedup for different application combinations and controller placements normalized to row $0-7$.

simulated in each simulation. We began statistics collection after the first 2 million cycles were complete. Relevant simulation parameters appear in Table I.

Although there are a total of $\left(\begin{array}{c}10 \\ 2\end{array}\right)$ different combinations of the applications, we present results for only 10 of these due to space constraints. We show the average over all combinations. In Figure 6, we present the weighted speedup [Snavely and Tullsen 2000] obtained for the different application combinations by the different designs. The speedup is normalized to that for the row 0-7 design. For the mesh network, mc-opt performs as well as diagonal and diamond designs. All of these designs have, on average, about $4.5 \%$ better performance than the row 0-7 design. For the torus network, mc-opt 


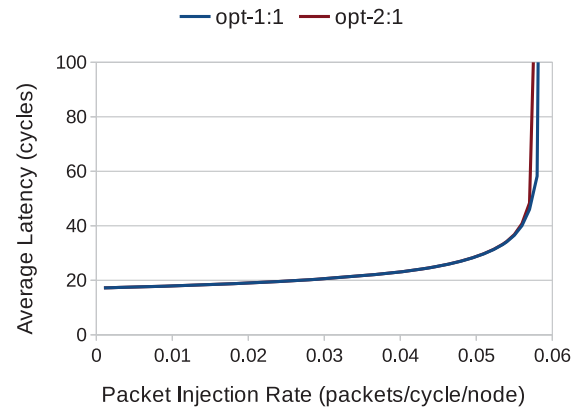

(a) Simulated traffic with read to write ratio $2: 1$

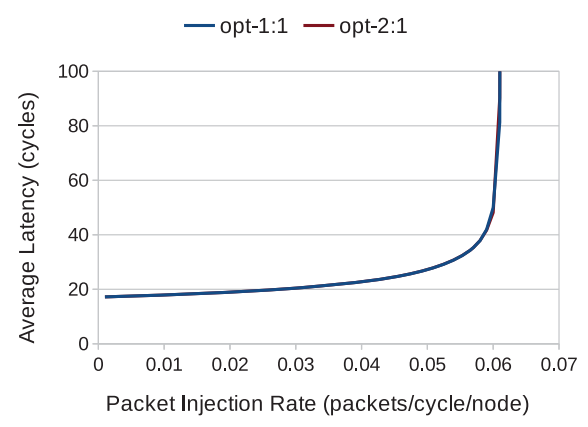

(b) Simulated traffic read to write ratio 1:1

Fig. 7. Average latency versus injection rate (uniform random traffic). (a) Traffic with read requests twice as probable as write requests. (b) Traffic with read and write requests being equiprobable. Both (a) and (b) show optimal designs obtained by solving the model. opt $-1: 1$ is for the setting $R=1$, and opt $-2: 1$ is for the setting $R=2$.

performs better than diagonal and diamond on almost all combinations. On average, mc-opt improves performance by slightly more than $1 \%$ over diagonal and diamond.

Thus, the solution obtained from the model works well even in real situations. We expect mc-opt to perform even better on workloads that have higher cache miss rates and hence access the main memory more frequently.

\subsection{Why MC-Opt Performs Better}

Fora torus network, mc-opt performs better than diamond and diagonal (which are isomorphic for this network). Our synthetic simulations show that mc-opt spreads out the traffic better. diagonal exhibits traffic hotspots around the clusters of four adjacent memory controllers, resulting in only $6.25 \%$ of the links observing at least $90 \%$ of the maximum channel load. In contrast, about $25 \%$ of links in mc-opt observe at least $90 \%$ of the maximum channel load. By more uniformly distributing memory controllers, mc-opt achieves more uniformly distributed traffic.

\subsection{Effect of Read and Write Traffic Ratio on Controller Placement}

To gauge the effect of the ratio of read versus write traffic on the placement of controllers, we also solved our optimization model for two more settings of parameters: ( $R=2, K=5$ ) and ( $R=10, K=5$ ). The first of these settings represents the typical ratio of $2: 1$ for reads to writes, whereas the second one is a rather extreme setting of $10: 1$ for the reads to writes ratio. A design obtained for read to write ratio $R: 1$ would be referred to as opt $-R: 1$. For example, the design with read to write ratio $2: 1$ is referred to as opt $-2: 1$.

We evaluated the designs obtained using the on-chip network simulator described earlier. We show the results in Figures 7 and 8. As can be seen in the figures, we observed almost no difference in performance of the designs opt $-1: 1$ and opt $-2: 1$. We believe that this is because the difference in the volume of traffic from cores to memory controllers and from memory controllers to cores is not that significant for the designs considered. We also observed that opt $-1: 1$ performs slightly worse than $o p t-10: 1$ on traffic with reads 10 times as probable as writes. But opt $-10: 1$ performs significantly worse on traffic with read to write ratio $1: 1$. This is because opt $-10: 1$ is optimized for the case when most of the traffic flows from memory controllers to cores. 


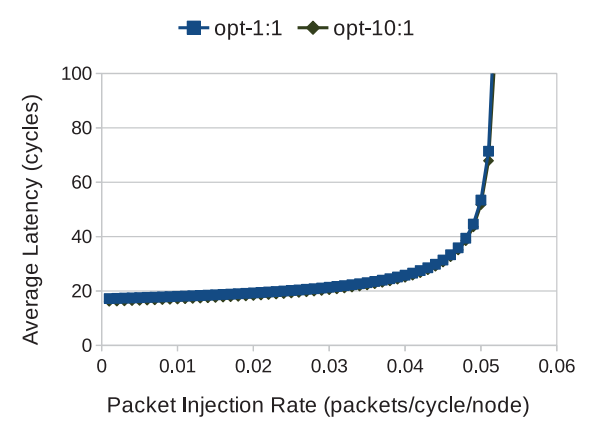

(a) Simulated traffic with read to write ratio 10:1

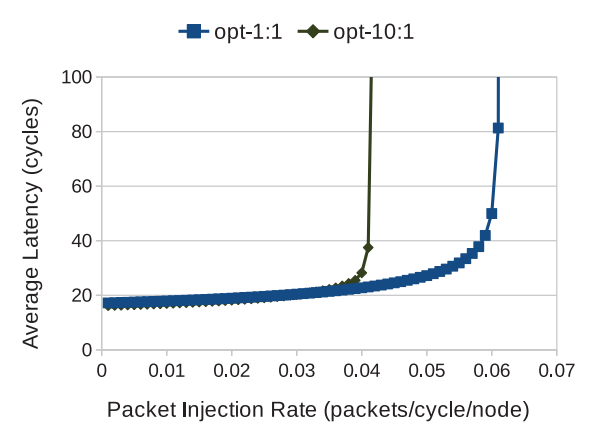

(b) Simulated traffic read to write ratio 1:1

Fig. 8. Average latency versus injection rate (uniform random traffic). (a) Traffic with read requests 10 times as probable as write requests. (b) Traffic with read and write requests being equiprobable. Both (a) and (b) show optimal designs obtained by solving the model. opt-1:1 is for the setting $R=1$, and opt $-10: 1$ is for the setting $R=10$.

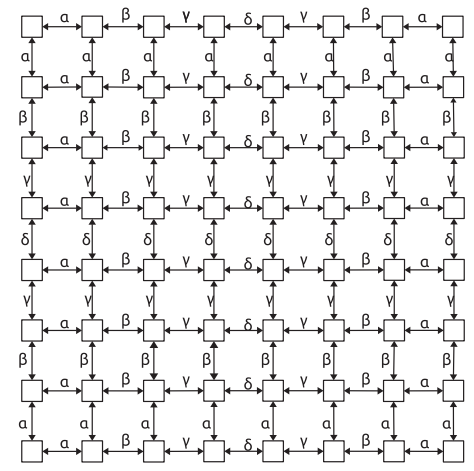

(a) Distribution of traffic on different links. Each link is marked with the amount of traffic it observes relative to the links that observe the maximum amount of traffic. $\alpha=0.4375, \beta=0.75, \gamma=0.9375, \delta=1$.

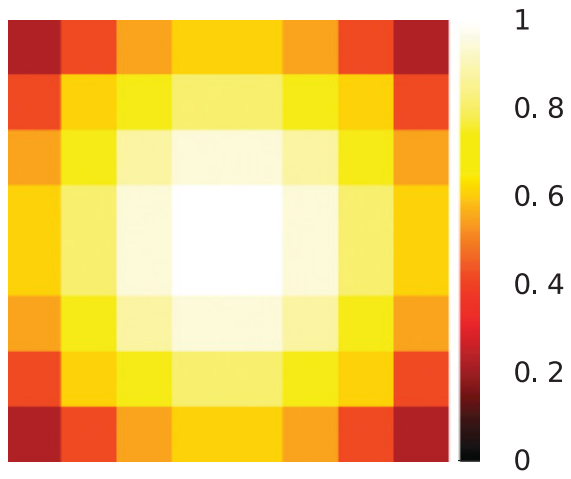

(b) Heatmap for traffic distribution in a mesh network un a uniform traffic assumption. Each cell represents a router. Its color represents the amount of traffic passing through the router.

Fig. 9. Traffic distribution in a mesh network.

\section{DESIGNING AN ON-CHIP NETWORK}

Mishra et al. [2011] observed that in an on-chip network based on a mesh topology, the routers and links closer to the center of the mesh handle more traffic than those near the edge of the mesh. This is represented pictorially in Figure 9. But the routers, whether at the center or at the edge, are typically provided with the same amount of buffers and virtual channels. Mishra et al. therefore raised the question on how the resources should be distributed across the routers, as it appears that allocating each router with the same resources may not be optimal. These observations were made assuming that the routing protocol is deterministic and dimension ordered, and that the traffic is uniformly distributed over all paths permissible under the routing protocol.

As an answer, Mishra et al. [2011] designed a heterogeneous mesh network composed of two types of links (wide and narrow) and two types of routers (big and small). To arrive at a distribution of these links and routers for an $8 \times 8$ mesh network, they evaluated several thousand design configurations for a $4 \times 4$ mesh network. The best 


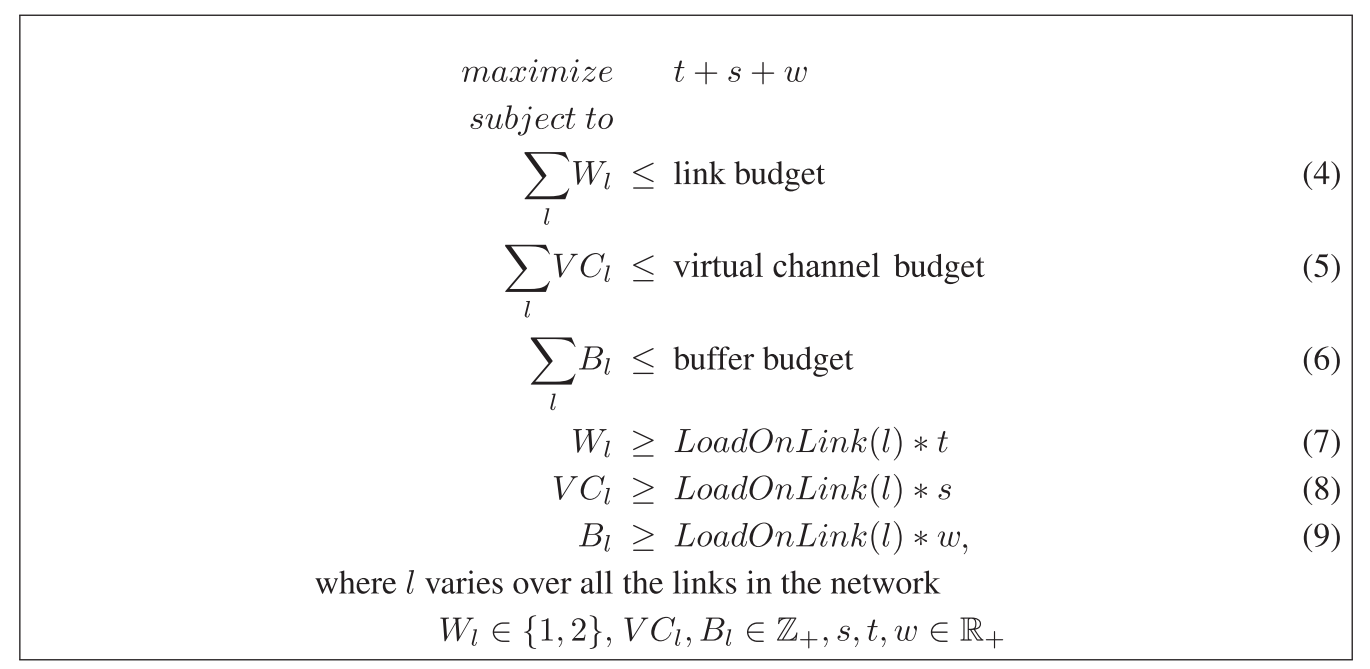

Fig. 10. MILP for distributing network resources.

configurations for the $4 \times 4$ mesh network were extrapolated to the $8 \times 8$ network. Later, Figure 12 shows the designs proposed by Mishra et al.

An $8 \times 8$ mesh network requires 64 routers. Assuming 16 big and 48 small routers, there are $\left(\begin{array}{c}64 \\ 48\end{array}\right) \approx 4.89 \times 10^{14}$ possible ways in which these routers can be placed in the network. If the assumption that routers can only be big and small is dropped, the solution space explodes further. Given the size of the solution space, we use mathematical optimization for solving this resource allocation problem. In a nutshell, the designer has a fixed budget of resources, and he or she needs to figure out the optimal resource division among the routers. The following sections describe an MILP for the problem.

\subsection{Assumptions Made in the Model}

To facilitate comparison, our optimization model relies on similar assumptions as Mishra et al. [2011]. The resources available for designing an on-chip network are links, virtual channels, and buffers. We can vary the bandwidth of the physical links connecting different routers, the number of virtual channels, and the number of buffers associated with each physical link. A physical link can be either wide or narrow. A wide link has twice the bandwidth of a narrow link. The sum total of the bandwidths of all links in the network is bounded by the link budget. This budget is only for the links between the routers. The links between the cache controllers and the routers are assumed to be thin. The total number of virtual channels and buffers across all routers are bounded by the virtual channel budget and the buffer budget, respectively. There is an upper bound on the number of virtual channels and buffers that can be associated with a physical link. Apart from these assumptions on the resources, the traffic distribution in the network is assumed to be known a priori.

\subsection{Notation Used in the Model}

Our model appears in Figure 10. For each unidirectional link $l$ in the mesh network, we introduce the following variables. $W_{l}$ is an integer variable denoting whether link $l$ is wide or narrow. A narrow link has width of 1 , whereas a wide link has a width of 2 . $V C_{l}$ is an integer variable denoting the number of virtual channels associated with the physical link $l . B_{l}$ is an integer variable for the number of buffers associated with link $l$. Variables $t, s$, and $w$ denote the bandwidth, virtual channels, and buffers allocated 
to a link that has a load of one unit. Last, the function LoadOnLink(l) gives the load on link $l$. This function is an input to the problem, as the traffic distribution is known beforehand.

\subsection{Description of the Model}

Now we describe our model in detail. Equations (4), (5), and (6) impose the budgetary constraints on the design. Intuitively, each link should have resources in proportion to the traffic that goes over that link. Such a distribution would allocate more resources to the routers at the center of the mesh network compared to the routers on the edge. Equations (7), (8), and (9) impose this proportionality constraint. We use the greater than or equal to $(\geq)$ relation in these constraints because $W_{l}, V C_{l}$, and $B_{l}$ are integer valued and the terms appearing on the right-hand side are nonnegative reals. The objective of the model is to maximize the sum: $t+s+w$. We chose this objective for two reasons:

-As mentioned previously, we would like to assign resources to each link in proportion to the traffic going over that link. In our model, variables $t, s$, and $w$ represent the constants of proportionality for the distribution of different resources. Equations (7), (8), and (9) ensure the proportionality constraints. But the resources, left after the proportional distribution, can be distributed arbitrarily. For example, if $t$, $s$, and $w$ are set to 0 , all resources can be distributed arbitrarily among the links. On the other hand, if these variables take the maximum value that they can possibly have, then almost all resources will be distributed proportionately.

- The model also minimizes the maximum amount of traffic handled by a unit amount of resource. Consider Equation (8). We can rewrite it as $\frac{1}{s} \geq \frac{\operatorname{LoadOnLink(l)}}{V C_{l}}$. Since $s$ is maximized, $\frac{1}{s}$ is minimized. Thus, the maximum load that a single virtual channel needs to bear is minimized.

From the model, it can be seen that $t, s$, and $w$ are independent of each other. Therefore, each of them will be independently maximized. In fact, the overall problem could be split into three independent optimizations.

\subsection{Design Obtained from the Model}

We solve the model under certain assumptions. The network is assumed to be an $8 \times 8$ mesh network, with dimension-order routing. Routers have up to six input and output ports. Two of these ports are for the private cache and the shared memory controllers, respectively. The other four ports are for the four different directions. Last, we assume that the traffic is distributed uniformly - that is, each cache controller generates requests for any of the memory controllers with equal probability. Thus, the traffic that a link needs to service is directly proportional to the number of paths using that link.

Figure 11 shows the design obtained on solving the model. Figure 11(a) shows the distribution of wide (256-bit) and narrow (128-bit) links across the $8 \times 8$ mesh network. All links that observe a heavy load (marked with $\gamma$ and $\delta$ in Figure 9(a)) are wide in our design. Figure 11(b) shows a heatmap for the distribution of the virtual channels among the routers. It can be observed that the number of virtual channels go down on moving from the center of the mesh toward the periphery. Since the traffic is assumed to be uniformly distributed, the routers at the center of the mesh service more traffic than those at the periphery, as shown in Figure 9(b). Therefore, the design has the most resources assigned to the routers at the center and the least to the ones farthest from the center.In the sequel, this design will be referred to as the net-opt design. 


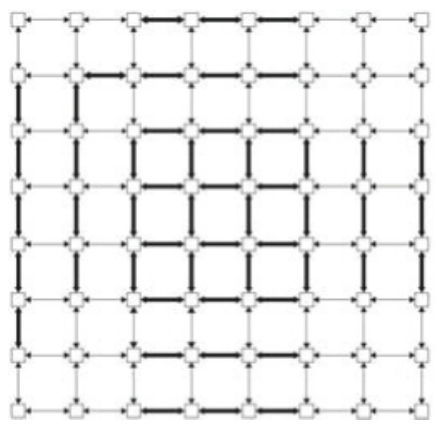

(a) Link width distribution. Wide links have been shown wider compared to narrow links.

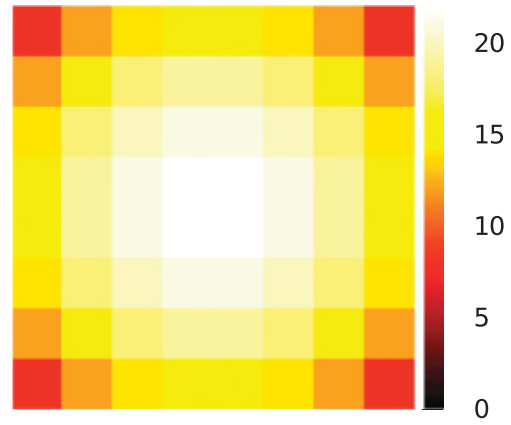

(b) Virtual channel distribution. Each cell represents a router. Its color represents the number of virtual channels assigned to the router.

Fig. 11. net-opt network design.

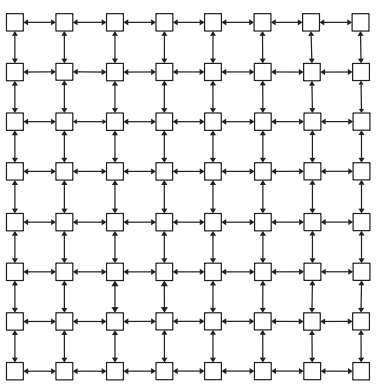

(a) base

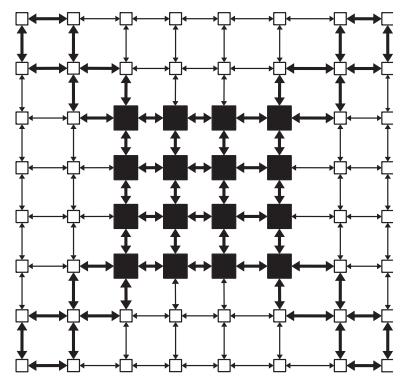

(b) center

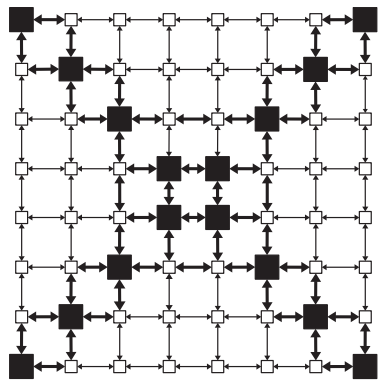

(c) diagonal

Fig. 12. Different network designs. Each box represents a router. Shaded routers are big. Wider links have been shown wider.

Note that there is slight asymmetry in the links in Figure 11(a). Wider links, other than those in the middle three rows and columns, were assigned more bandwidth because of the available budget. If symmetry is important (e.g., to simplify layout), it is straightforward to add symmetry constraints.

\subsection{Evaluation of the Design}

We compare our design against the three designs analyzed by Mishra et al. [2011]. These designs have been shown in Figure 12:

—base: All routers are provided equal resources. Each port has three virtual channels per virtual network. Each link is 192 bits wide.

- center: Sixteen routers in the center are big (six virtual channels/virtual network/port). The rest are small (two virtual channels/virtual network/port). Half the links are wide (256 bits), whereas others are narrow (128 bits) as shown in Figure $12(\mathrm{~b})$.

—diagonal: Routers along the diagonals are big and the rest are small. Again, half the links are wide and others are narrow. The design appears in Figure 12(c). Mishra et al. [2011] found this design to be the best.

All designs in our experiments adhere to the same resource limits. Further, we assume that base operates at a clock frequency of $2.20 \mathrm{GHz}$ and all other designs operate at 


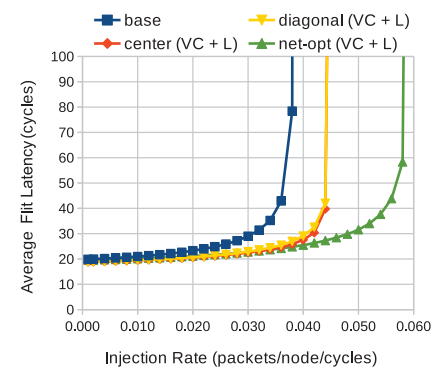

(a) Uniform traffic

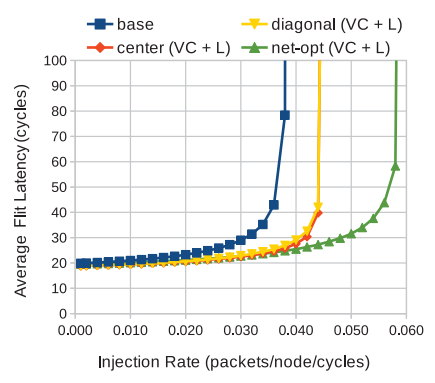

(b) Tornado traffic

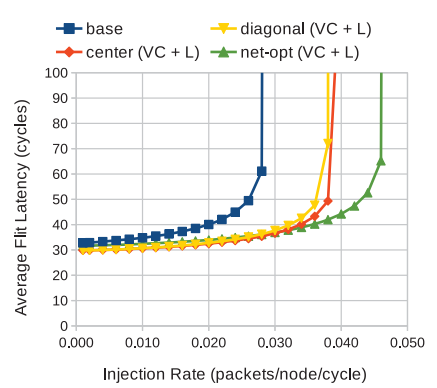

(c) Bit-complement traffic

Fig. 13. Average latency versus request injection rate for different request patterns.

2.07GHz. This assumption is required, as the routers in center, diagonal, and net-opt are possibly bigger than the routers in the base design. The frequencies assumed are same as those assumed by Mishra et al. [2011]. Since net-opt makes use of routers that are smaller than the big routers in center and diagonal designs, our assumption on the frequency of these routers should be sufficient.

4.5.1. Performance with Synthetic Traffic. We evaluate the candidate designs using the network simulator available in gem5 [Binkert et al. 2011]. The standard simulator supports a homogeneous network in which each physical link has the same width and the same number of virtual channels. We modified the simulator so that different links can be assigned a different number of virtual channels and link widths.

In each simulation, the L2 controllers inject request packets into the network at a fixed rate. A memory controller, on receiving a request, injects the response packet for that request, thus completing the request-response loop. The simulation is allowed to run 2 million cycles. At the end of the simulation, we note the average latency involved in transporting a flit from its source to the destination. The experiment is repeated with different rates of request injection and with three different request patterns-uniform random, tornado, and bit complement [Dally and Towles 2003].

Figure 13 shows the graphs for the average latency of a flit versus the rate of request injection into the network for these request patterns. As can be seen in the graphs, for all three request patterns, the net-opt design has a higher saturation bandwidth and provides lower average latency compared to all other designs. This experiment shows that the design obtained from the optimization-based model works well when a detailed network simulation is carried out, even when the uniformity assumption for the traffic is not observed. net-opt performs better than diagonal and center because the latter two designs distribute resources better than base but do not do so completely. In particular, all routers and links in rows and columns 2 , 3,4 , and 5 of the mesh network observe more traffic than rest, as shown in Figure 9. net-opt provides the most resources to these routers and links. In contrast, diagonal and center provide more resources to routers and links lying along the diagonals.

With the same setup as described earlier, we also simulated network designs that use a lesser amount of link resources by reducing the number of wider links. In Figure 14, we show how the peak sustained bandwidth varies as a function of the amount of link resources. As we can see from the figure, optimal distribution of the link resources provides for similar or better performance even when the number of wider links used in the design is $30 \%$ less compared to diagonal and center. 


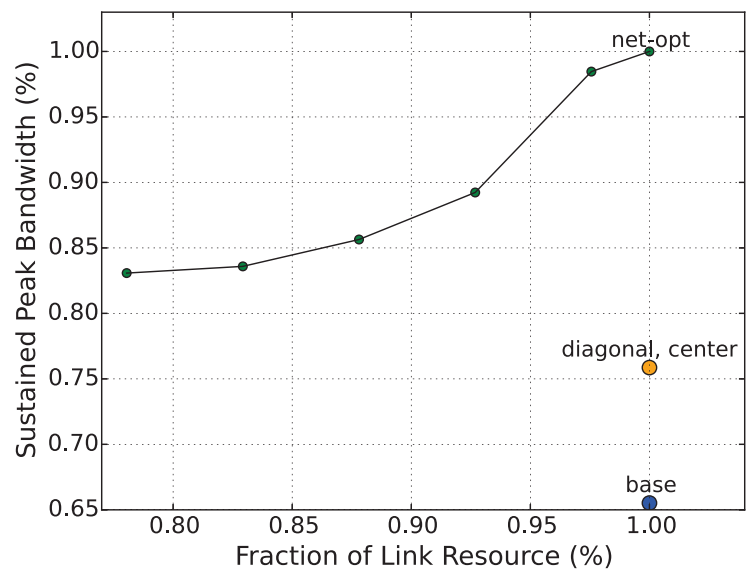

Fig. 14. Sustained peak bandwidth as a function of the link resources used.

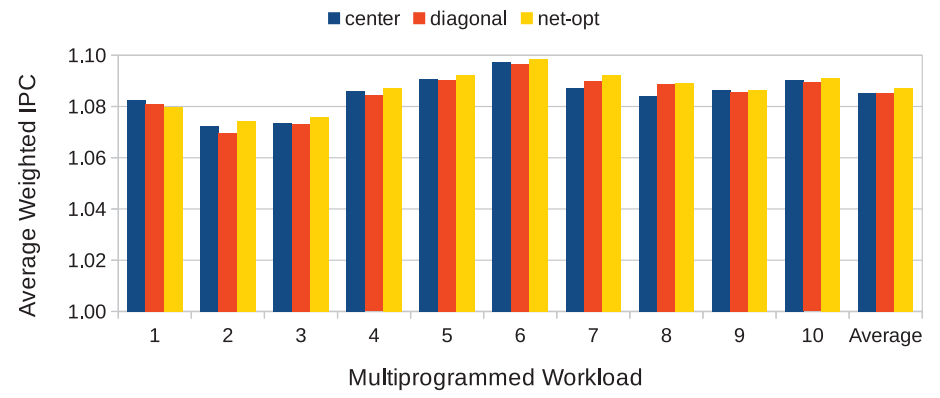

Fig. 15. Weighted speedup for different application combinations and network designs normalized to base.

4.5.2. Performance with SPEC CPU2006 Applications. We further evaluated the designs by simulating applications from the SPEC CPU2006 benchmark suite. The experimental setup was described in Section 3.5.2. In Figure 15, we present the weighted speedup [Snavely and Tullsen 2000] obtained for the different application combinations by the different designs. The speedup is normalized to that for the base design. net-opt performs as well as diagonal and center designs. All of these designs have, on average, about $8 \%$ better performance than the base design. net-opt fails to perform better than diagonal and center, as the network utilization is very low, and all designs observe nearly zero load latency for all the workloads.

\section{ON-CHIP NETWORK DESIGN COMBINED WITH PLACEMENT OF CONTROLLERS}

The model in the previous section assumes the traffic distribution as an input. But this distribution depends on the placement of the memory controllers, which in turn may depend on the network design. Ideally, we would like to place memory controllers and allocate network resources in a single combined problem. This may result in a better design than that obtained by solving the two problems sequentially. With this intuition, we formulated the optimization model presented in Figure 16.

\subsection{Analysis of the Model}

Most of the variables and the constraints used in the model have been described in Sections 3 and 4 . Now we describe the additional variables and constraints: 


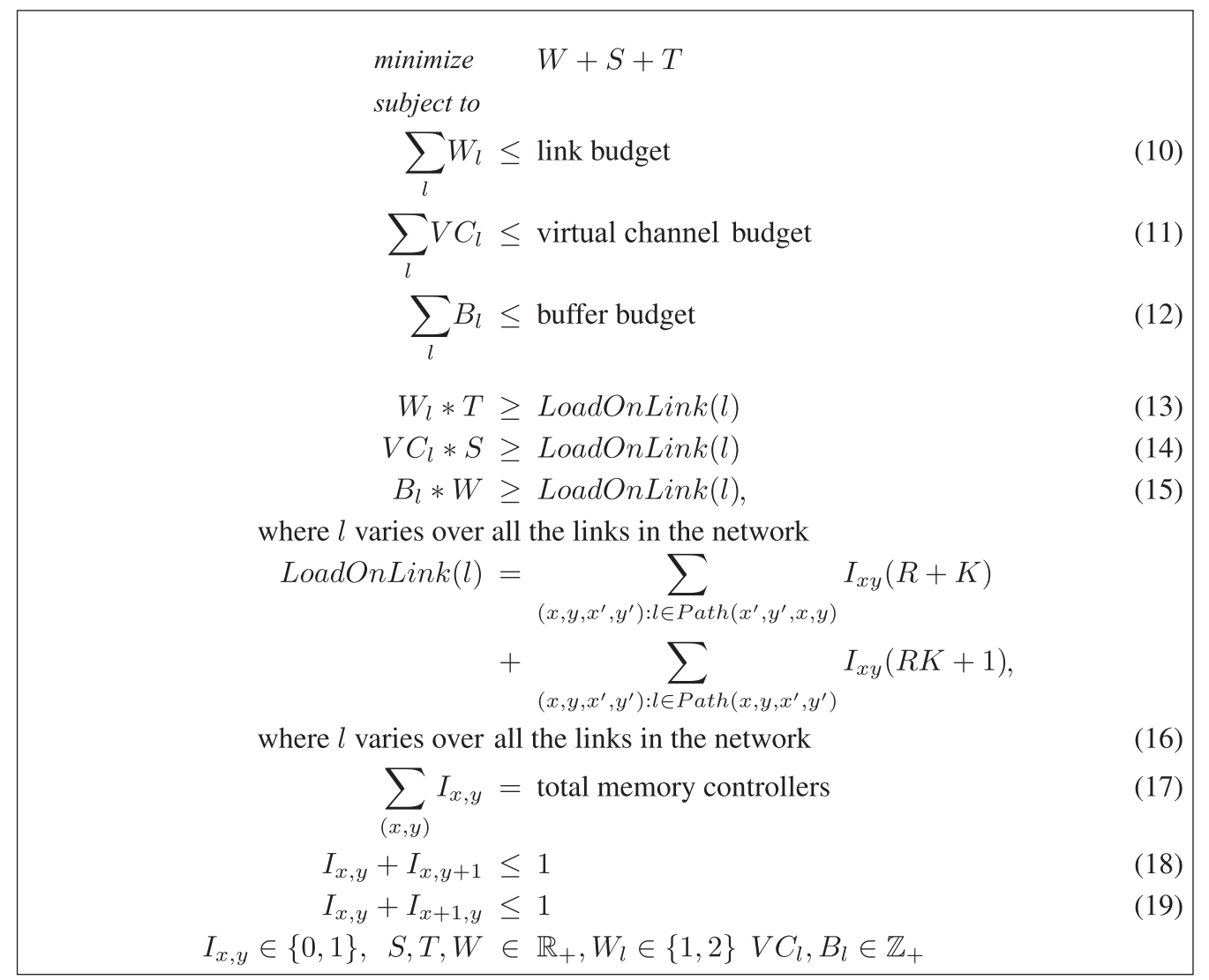

Fig. 16. Nonlinear program for the combined problem.

-Variables $T, S$, and $W$ represent the load per unit bandwidth, virtual channel, and buffer, respectively. They are the inverses of the variables $t, s$, and $w$ introduced in Section 4.2. Note that although $t, s$, and $w$ can be independently optimized, $T, S$, and $W$ cannot be since they are linked by variables $I_{x y}$.

-Constraints (18) and (19) together avoid designs in which adjacent cells in the mesh network have memory controllers. We observed that without these (or similar) constraints, all memory controllers are placed in the center portion of the chip. Such a design is likely to cause congested wire routing and thermal hot spots, hence the additional constraints in the model.

- Since memory controller placement determines traffic patterns, the function LoadOnLink $(l)$ is no longer an input. In addition, we assume that caches are private to the cores and that the on-chip network is only used for communication between the last-level (private) caches and the memory controllers. Therefore, constraint (16) only accounts for traffic to and from memory controllers.

- Constraints (13), (14), and (15) have terms where two variables are being multiplied. These product terms make these constraints nonlinear. Hence, the model is an MINLP.

- The reasons behind the choice of objective function are similar to the ones discussed in Section 4.3. 


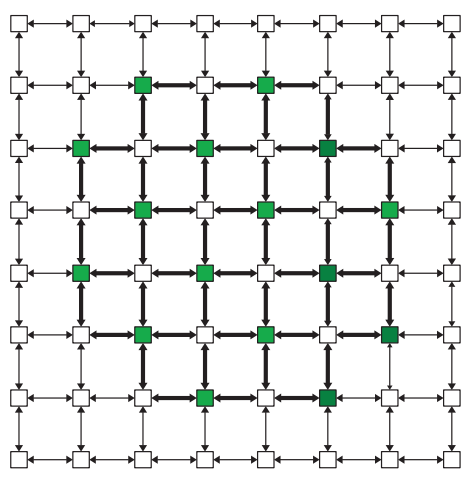

(a) Distribution of memory controllers and link widths

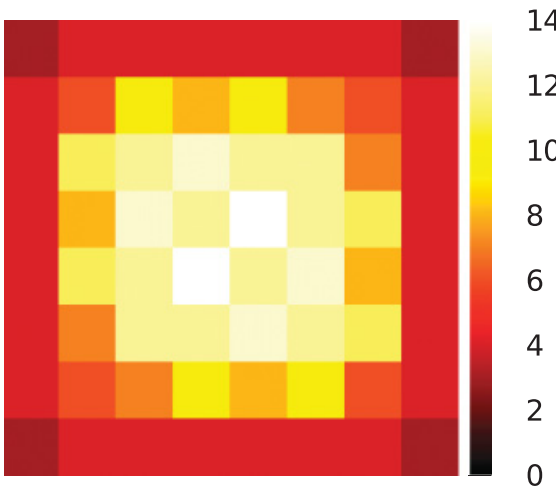

(b) Virtual channel distribution

Fig. 17. com-opt design for the combined problem. (Reproduced from Nowatzki et al. [2013] with permission from Morgan \& Claypool Publishers.)

Our model is nonconvex for two reasons. In our model, variables $W_{l}, V C_{l}, B_{l}$, and $I_{x, y}$ are constrained to be integer valued and models with integrality constraints are nonconvex. Even if we were to drop the integrality requirements, the model remains nonconvex because constraints (13), (14), and (15) are not convex. These three constraints are of the form $f(x, y, z)=z-x y \leq 0$. If we choose, for example, $a=(1,1,1)$ and $b=(2,2,4)$, then $f(a)=f(b)=0$ but with $\theta=0.5, f(\theta a+(1-\theta) b)=0.25$ so that

$$
f(\theta a+(1-\theta) b) \leq \theta f(a)+(1-\theta) f(b)
$$

is not satisfied. Thus, $f$ is not convex, implying that constraints (13), (14), and (15) and the overall model are not convex.

\subsection{Solving the Model}

We made several assumptions for solving the model. The network was assumed to be an $8 \times 8$ mesh network with dimension-ordered routing and uniformly distributed traffic.

No polynomial-time methods are known for solving nonconvex MINLPs [Boyd and Vandenberghe 2004]. We explored the solution space for our problem using Baron [Tawarmalani and Sahinidis 2005], an NLP solver. The solver uses a branching scheme to optimize over the (bounded) feasible set. A good initial solution is quite useful for pruning the search tree to find the globally optimal solution. To get closer to the optimal design, we seeded the solver with multiple initial designs and experimented with the bounds on different variables. We used the placements described in Section 3 as initial designs for the solver. The solver ultimately computed designs with improved objective function values. Figure 17 shows the best design we found.

As we show in Section 5.3, this is in fact the globally optimal solution. However, in the given time limit, Baron could not prove the global optimality of this solution. Rather, by relaxing the nonconvex constraints and iteratively performing a branching procedure, it provides a lower bound on the optimal value of the objective function. The design shown in Figure 17 has an objective value that is $14 \%$ higher than the algorithmically computed lower bound. In comparison, the design of Mishra et al. [2011] (shown in Figure 18) has an objective value that is 55\% higher than the lower bound. 


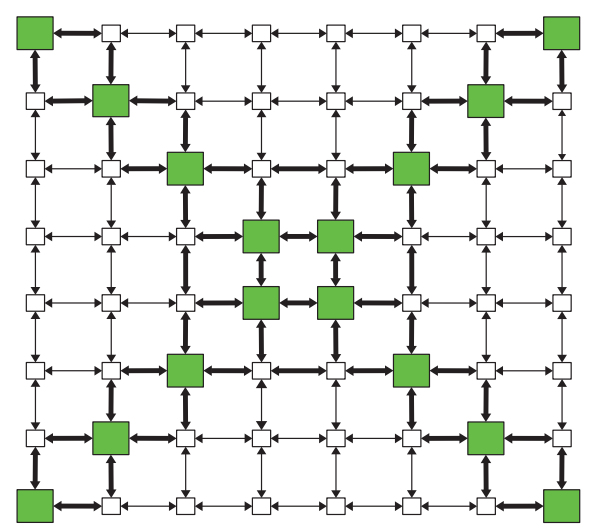

Fig. 18. Distribution of memory controllers, buffers, and link widths for diagonal. (Reproduced from Nowatzki et al. [2013] with permission from Morgan \& Claypool Publishers.)

\subsection{Linearized Model}

Our initial model has a linear objective function and nonlinear constraints with the form $f(x, y, z)=z-x y \leq 0$. These are called bilinear terms, as each is the product of two variables, and the whole model is called a bilinear program (BLP). Moreover, each bilinear term is the product of a continuous variable and an integer variable, which can be converted to linear terms using binary expansion [Gupte et al. 2012]. After this conversion, we can solve the model using MILP techniques.

In our model, constraints (13), (14), and (15) are of the form $z \leq x y$, where $x$ is a continuous variable and $y$ is an integer variable. Further, both $x$ and $y$ are nonnegative and bounded from above (i.e., $0 \leq x \leq a$ and $0 \leq y \leq b$ ). The set of points satisfying such a constraint can be represented as

$$
\mathcal{P}=\left\{(x, y, z) \in \mathbb{R}_{+} \times \mathbb{Z}_{+} \times \mathbb{R}: z \leq x y, x \leq a, y \leq b\right\} .
$$

Using $y$ 's binary expansion, we get $y=\sum_{i=1}^{k} 2^{i-1} w_{i}$. Here, $w_{i}$ are $0-1$ integer variables and $k=\left\lfloor\log _{2} b\right\rfloor+1$. Define $\mathcal{B}$ as

$$
\begin{array}{r}
\mathcal{B}=\left\{(x, y, z, w, v) \in \mathbb{R} \times \mathbb{Z} \times \mathbb{R} \times\{0,1\}^{k} \times \mathbb{R}^{k}:\right. \\
y=\sum_{i=1}^{k} 2^{i-1} w_{i}, y \leq b, z \leq \sum_{i=1}^{k} 2^{i-1} v_{i}, 0 \leq v_{i} \leq a w_{i}, \\
\left.v_{i} \leq x, v_{i} \geq x+a w_{i}-a, \text { for all } i \in\{1, \ldots, k\}\right\} .
\end{array}
$$

It can be shown that $\mathcal{P}=\operatorname{Proj}_{x, y, z}(\mathcal{B})$. Here, $\operatorname{Proj}_{x, y, z}$ represents the projection operator that maps $(x, y, z, w, v)$ to $(x, y, z)$. Since $\mathcal{B}$ does not have any nonlinear term in its representation, it is an exact linearization of $\mathcal{P}$. We linearize our model by replacing constraints (13), (14), and (15) with the constraints used in defining $\mathcal{B}$. With this new model, it took CPLEX [IBM Decision Optimization 2015], another solver for MILP, less than 5 minutes to prove that the design presented in Figure 17 is in fact optimal.

\subsection{Optimal Design}

Figure 17 illustrates the design-referred to as com-opt-obtained from solving the model. Figure 17(a) shows the distribution of the memory controllers (solid boxes) and 
the wide (bold) and narrow (thin) links in the network. Figure 17(b) shows a heatmap indicating the distribution of virtual channels. Note the significant differences from the optimal solutions to the individual problems in Figures 4(e) and 11.

We also obtained the design center-opt as the solution to our model from Figure 16 without constraints (18) and (19). In this design, the memory controllers are placed in the center of the chip. The objective value for center-opt is better than that of com-opt by $1.25 \%$. We therefore expect center-opt to perform marginally better than com-opt.

\subsection{Sensitivity of the Optimal Design vis-à-vis the Objective Function}

The objective function in the combined model essentially gives equal consideration to links, virtual channels, and buffers. To test the sensitivity to this assumption, we generalized the objective function to provide weights to the variables $S, T$, and $W$. Specifically, the objective from Figure 16 was changed to $\omega W+\psi S+\tau T$. We considered the following cases:

(1) $\omega=\psi=\tau=1$ : This case has been evaluated in Section 5.4, and com-opt was obtained as the optimal design for the model.

(2) $\omega=2, \psi=\tau=1$ : The design com-opt is optimal for this setting as well.

(3) $\omega=1, \psi=2, \tau=1$ : com-opt was found to be optimal.

(4) $\omega=10, \psi=1, \tau=1$ : com-opt was found to be optimal.

(5) $\omega=1, \psi=10, \tau=1$ : com-opt was the best design found, but the solver CPLEX was not able to prove that it is the optimal design. The optimality gap (i.e., the gap between the objective of the best design found and the best underestimate) was $18 \%$.

\subsection{Evaluation of the Designs}

The objective function value for com-opt and center-opt is better compared to that for the design proposed by Mishra et al. [2011]. Their design, referred to as diagonal and shown in Figure 18, places memory controllers on the diagonal nodes, along with big routers (six virtual channels/port) and wide links. Routers on nondiagonal nodes are small (two virtual channels/port) and use narrow links. To validate this result, we compare these designs using simulation. We also evaluate two other designs: row0$7+o p t$ and diamond+opt. The placement of memory controllers for these is shown in Figure 4. The distribution of link widths and virtual channels was obtained by solving our optimization model with variables for memory controllers $\left(I_{x, y}\right)$ set to fixed values. All designs use the same number of virtual channels, wide and narrow links, and buffers.

5.6.1. Synthetic Traffic. We evaluated the designs using the network-on-chip (NoC) simulator described in Section 4.5.1. In Figure 19(a), we present the average latency experienced by a flit as a function of the rate of request injection into the network. From the graph, we can observe that com-opt, center-opt, and diamond+opt support about $30 \%$ higher saturation bandwidth and provide lower latency compared to diagonal.

5.6.2. SPEC CPU2006 Benchmark. We also evaluated the designs by simulating combinations of SPEC CPU2006 benchmarks [Henning 2006] on the gem5 simulator as described in Section 3.5.2. In Figure 19(b), we present the weighted speedup obtained for the different combinations. The speedup is normalized to the diagonal design. It can be observed that, on average, com-opt improves weighted speedup by $5.4 \%$ and center-opt improves weighted speedup by $6.7 \%$ over diagonal.

5.6.3. NAS Parallel Benchmarks. We further evaluated the designs by executing applications from NPB [Bailey et al. 1991]. There are eight applications that comprise NPB. We provide results only for the ones that gem5 can execute properly. Others fail due to lack 


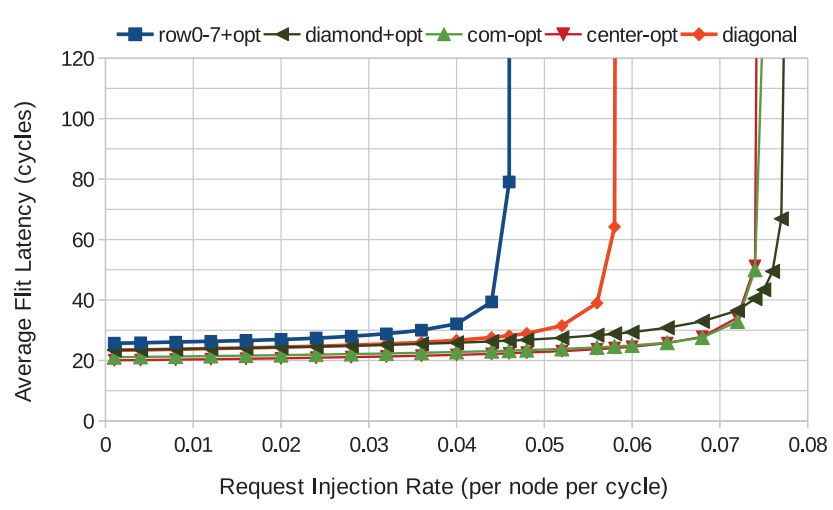

(a) Graph for Average Flit Latency vs Request Injection Rate for synthetically generated uniform random traffic.
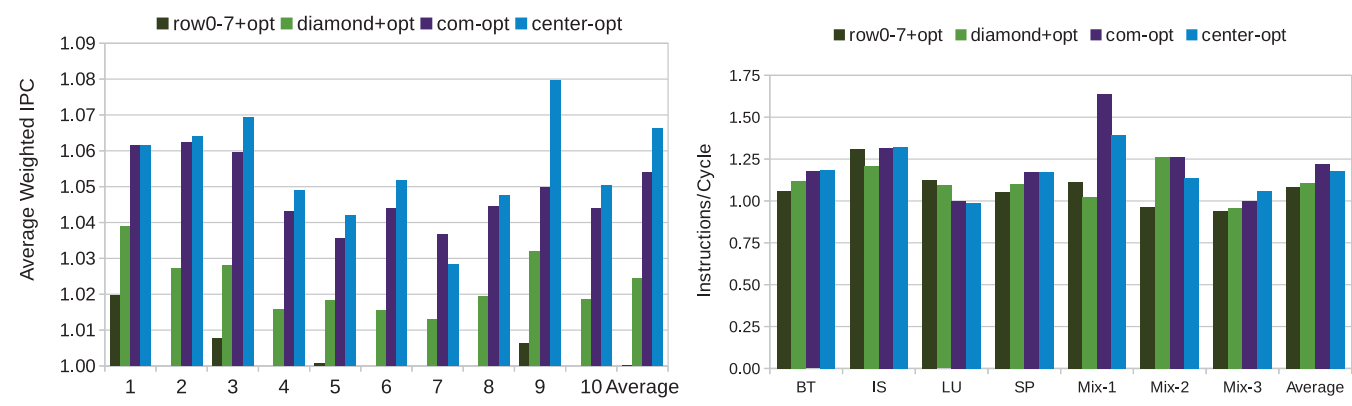

(b) Weighted speedup, normalized to diagonal for multiprogrammmed workloads composed from SPEC CPU2006 applications.

(c) Instructions per cycle, normalized to diagona I for multithreaded workloads composed from NAS Parallel Benchmarks.

Fig. 19. Graphs from experimental evaluation of designs for the combined problem.

of support for x87 instructions in gem5. Each application is executed with four threads running on adjacent cores. Thus, we ran 16 applications for a particular simulation to cover all 64 cores. We also ran some workloads with a mix of these applications. The applications were mapped randomly to the cores for such workloads. Note that in these simulations, the on-chip network traffic is not uniformly distributed, as the cache-to-cache transfers take place among caches private to cores executing threads from the same application.

We executed each workload five times with different random seeds to harmonize any effects arising due to scheduling of threads. In Figure 19(c), we present the improvement in IPC obtained for different designs. The speedup is normalized to the diagonal design. It can be observed that com-opt performs about $22 \%$ better than diagonal.

\subsection{Analysis of the Designs}

com-opt performs better than diagonal for two reasons:

-The zero load latency of com-opt is slightly lower than that of diagonal, as illustrated in Figure 19(a). Hence, under low traffic intensity, com-opt results in lower latency. 


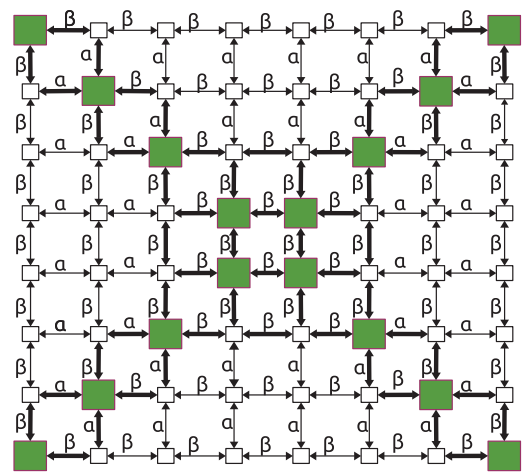

(a) Traffic load on different links. Links marked $\alpha$ are lightly loaded, whereas links marked $\beta$ are heavily loaded.

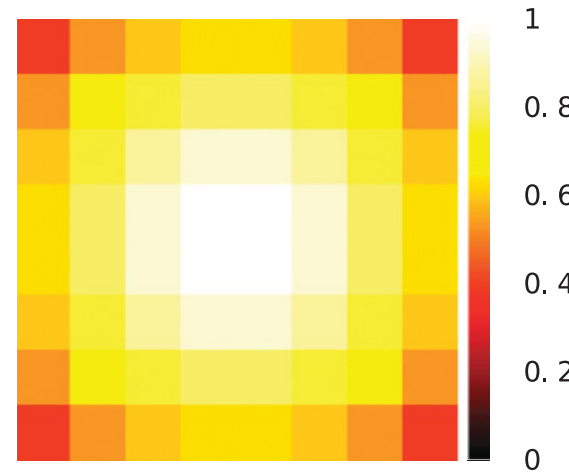

(b) Per router traffic load

Fig. 20. Traffic distribution for the diagonal design.

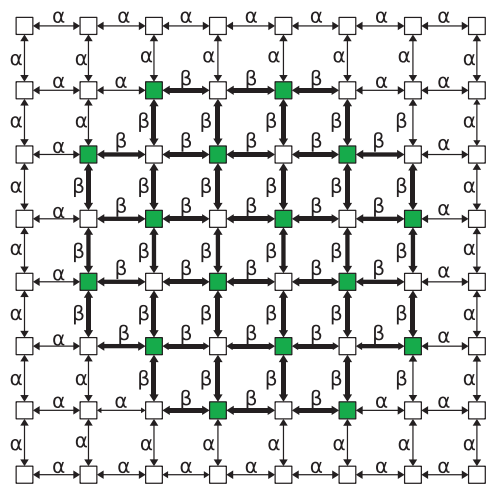

(a) Traffic load on different links. Links marked $\alpha$ are lightly loaded, whereas links marked $\beta$ are heavily loaded.

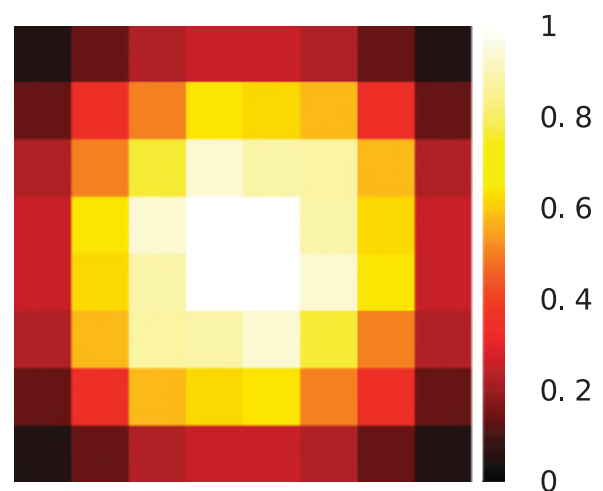

(b) Per router traffic load

Fig. 21. Traffic distribution for the com-opt design.

-For higher traffic intensity, com-opt performs better, as it does a better job of matching network resources to network traffic. Figures 20 and 21 illustrate the traffic load observed by the links and the routers for the two designs. Links marked $\alpha$ observe less traffic compared to links marked $\beta$. Ideally, the $\alpha$ links should be narrow and the $\beta$ links should be wide. But diagonal has 16 wide $\alpha$ links and 32 narrow $\beta$ links, indicating a mismatched resource allocation. Similarly, diagonal assigns more virtual channels to routers near the corners even though they observe less traffic. By simultaneously placing memory controllers and allocating network resources, com-opt eliminates these resource mismatches.

\section{IMPROVING THE MODELS}

The design problems that we have discussed have several possible variations that can be handled without significant changes to the approach presented. The formulations provided can handle different types of resources, routing protocols, and topologies. But there are other dimensions along which the models can be improved to better relate with actual designs. 


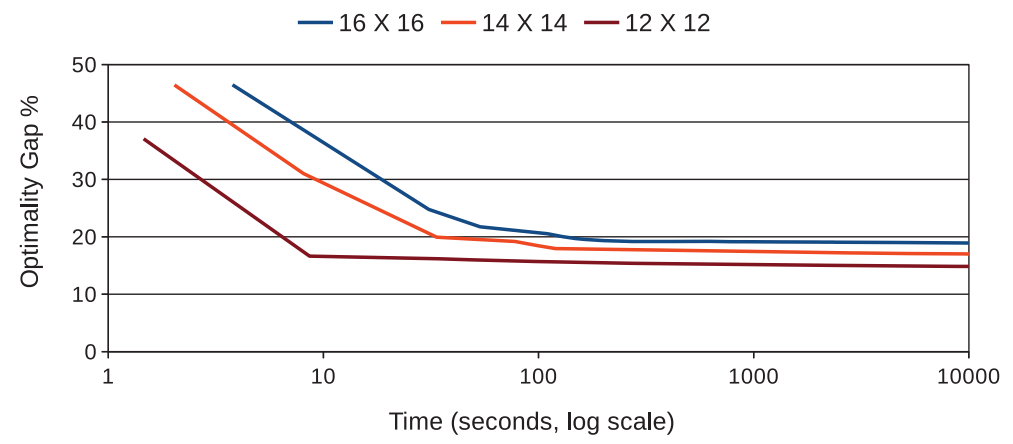

Fig. 22. Reduction in optimality gap as a function of time. An $n \times n$ problem refers to a problem with $n^{2}$ processors and $2 n$ memory controllers. Optimality gap is the percentage difference in the objective value for the best design found and the best underestimate.

The models presented in this article optimize the bandwidth available for on-chip traffic, following the lead of Abts et al. [2009]. However a different objective function may provide a better design point. As seen in Figures 5, 13, and 19, even though our designs provide much higher peak bandwidth, applications do not substantially benefit from the improved bandwidth. This indicates that average delay may be a better objective, as many applications tend to be latency sensitive. A major part of delays in an on-chip network is the serialization delay, which depends on the links' bandwidth. Since we allocate bandwidth to links in our models, a model optimizing for average delay should account for changes to serialization delay. One may also use concepts from theory of queueing systems [Bertsekas and Gallager 1992] for modeling average delay.

Our models assume that on-chip traffic is uniformly distributed. If the traffic is not distributed uniformly, a weighting function should be introduced to associate a weight with each path. For example, in asymmetric designs, it is likely that the traffic distribution would not be uniform. A subset of cores and memory controllers may observe more traffic than the rest. Formulations would have to be adapted to take care of the asymmetry. In addition, our models do not account for the effect of the cache hierarchy on the network traffic. Recent work on accurately modeling traffic distributions [Bogdan et al. 2010; Bogdan 2015] may be leveraged to improve the models. In design problems with time-varying traffic distributions, designing for multiple different traffic distributions using a stochastic optimization-based approach is also conceivable.

The formulations can also be extended by adding constraints on the power/energy consumed by the designs and thermal constraints that seek to avoid hot spots on the chip.

\section{WHY USE OPTIMIZATION?}

Mathematical optimization, as a performance analysis tool, has several potential advantages over other approaches.

\subsection{Scalability}

For the memory controller placement problem, the GA approach of Abts et al. [2009] required running heuristic-based algorithms on multiple machines over more than a day [Gibson 2012].Our approach found an optimal solution to the 64-processor, 16-port problem in less than a minute on a four-core, eight-thread machine. The same machine took less than 15 minutes to solve the 100-processor, 20-port problem. For bigger design problems, we were not able to prove the optimality of our solutions. In Figure 22, we 
show how the gap between the best design found and the best underestimate (defined in Section 2) for these design problems goes down with time. In each case, designs close to the estimated lower bound were found quickly. But closing the gap further would require a better model.

Similarly, Mishra et al. [2011] evaluated only several thousands of the possible $4 \times 4$ mesh network designs to arrive at what they thought was the best design for an $8 \times 8$ mesh network. Evaluating each design took 5 to 10 minutes, requiring multiple machines in parallel to reduce the total time [Mishra 2012]. Since the design space is huge, exploration via exhaustive/randomized simulation is impractical. In comparison, our optimization-based model took less than a minute for computing the design that is optimal under the given constraints.

Thus, mathematical optimization can potentially reduce the time required for design space exploration and is better suited for exploring larger and more complex design problems.

\subsection{Flexibility}

Extrapolation and divide-and-conquer-based approaches require the design problem to be symmetric. These approaches use the symmetry to reduce the complexity of the solution space. For example, the methods used by Abts et al. [2009] and Mishra et al. [2011] required the on-chip network to have the same dimension along the $X$ and the $Y$ axes. This was because the authors were trying to extrapolate results from a smaller $4 \times 4$ on-chip network to a larger $8 \times 8$ on-chip network. Although optimization-based models also benefit from using symmetry, given the speed of computation, it may not be necessary to restrict the design space to symmetric designs only. For example, in the optimization-based model, the $X$ and the $Y$ dimensions can differ. Similarly, it is not required that the number of memory ports be an integral multiple of the dimensions of the mesh/torus network. This was required in the original approach, again because of the need for extrapolation.

\section{LIMITATIONS OF OPTIMIZATION}

Despite the benefits, mathematical optimization may not be applicable to a large set of computer architecture problems. Optimization requires that the problem be represented using algebraic functions, which is not always possible. Many design problems require detailed models that may not be suitable for optimization techniques.

Additionally, optimization-based models may face computational challenges in exploring the design space. MILPs are known to be NP-hard [Papadimitriou 1981], so it is not always possible to obtain an optimal solution in an acceptable time frame, limiting its use on time constrained operational problems. No practical algorithms are known for solving problems involving nonlinear, and/or nonconvex function, such as $E D$ or $E D^{2}$, to global optimality [Boyd and Vandenberghe 2004]. Available solvers use different heuristics that might result in high computational effort or suboptimal solutions.

Moreover, many problems in computer architecture require performing a trade-off among multiple objectives. For example, one may need to design an on-chip network where both the network bandwidth and the network latency need to be optimized. For such problems, one may have to solve multiple instances of the optimization model to trace out a Pareto frontier. This is only possible if solving individual instances is fast enough. Otherwise, one may have to weight the different objectives based on expert opinion.

Finally, a given solution is only optimal for the specific instance of the model. Changes to inputs or the model's structure may make the solution suboptimal or infeasible. One may have to use techniques like stochastic optimization [Shapiro et al. 2009] if only 
probability distributions are known for the input parameters or robust optimization when only bounds on data uncertainty are given.

\section{RELATED WORK}

We have made a case for using mathematical optimization for solving design problems in the field of computer architecture. Our work is highly influenced by the work of Abts et al. [2009] and Mishra et al. [2011]. In this section, we highlight other recent work in the area and discuss why our approach is different.

On-chip networks. Designing on-chip networks is a fertile area of research. Significant effort has been devoted toward improving the performance and reducing the cost of on-chip networks. Prior work has proposed adaptive routing [Ma et al. 2011, 2012], bufferless NoC [Hayenga et al. 2009; Moscibroda and Mutlu 2009], and QOS support for NoCs [Grot et al. 2011]. These approaches alter the dynamic behavior of the network. Ben-Itzhak et al. [2012] proposed using simulated annealing for designing heterogeneous NoCs. We discussed earlier that mathematical optimization works better when the problem can be expressed as a linear/integer linear/convex program. Our work shows that this is true for certain problems related to NoC design. Heuristic-based approaches may work better for nonconvex design problems. Jang et al. [2015] analyzed traffic patterns for GPGPU applications partitioning schemes for virtual channels that improve resource utilization for on-chip networks. They also evaluated how these schemes interact with different routing protocols and memory controller placements.

Mathematical optimization has been used by other researchers to solve on-chip network design problems. Kinsy et al. [2009] used an MILP-based approach for producing deadlock-free routes. Abdel-Gawad and Thottethodi [2011] proposed program transformations for more efficient stream communication. These transformations are modeled using MILP solved at compile time. Srinivasan et al. [2004] presented an MILP-based approach for designing on-chip networks with homogeneous routers. The problem that we study looks at distributing link bandwidths, virtual channels, and buffers to heterogeneous routers. We also combine the heterogeneous network design and memory controller placement problems together, which has not been solved using MILP in the past.

On-chip placement. Prior work [Awasthi et al. 2010; Wang and O'Boyle 2009] focused on figuring out the best mapping for applications and data onto cores and memory controllers at execution time, whereas we present a design time approach. A lot work on on-chip placement has been done in the system-on-chip domain. These works [Zhou et al. 2006; Hung et al. 2005] proposed using GAs for generating solutions. Xu et al. [2011] also tackled the problem of placing memory controllers for CMPs. They solved the problem for a $4 \times 4 \mathrm{CMP}$ through exhaustive search. To find the best placement for the $8 \times 8$ problem, they exhaustively searched through solutions obtained by stitching solutions obtained for the $4 \times 4$ problem. This approach reduces the solution space that needs to be searched, but the idea is not generic. It assumes that the chip can be divided into smaller regions and that solutions for the smaller regions can composed to generate optimal solutions for larger regions. This may not hold true in general. Our approach of using mathematical optimization does not rely on any such assumption.

Theory. The network design problem has been widely studied in theoretical computer science [Magnanti and Wong 1984; Goemans et al. 1994], particularly with respect to designing distribution, transportation, telecommunication, and other types of networks. These works mainly focused on designing approximation algorithms for the different variants of the network design problem and on analyzing their theoretical complexity. We focus on on-chip network design and on-chip placement. 


\section{CONCLUSION}

In this article, we solved three problems related to on-chip networks. The solutions were obtained by using mathematical optimization models for searching and pruning the design space. We believe that there are other computer architecture problems that can benefit from optimization. It might be worthwhile to explore whether there are optimization techniques that are better suited to problems in computer architecture.

\section{ACKNOWLEDGMENTS}

We thank the editors, the anonymous reviewers, and members of the Multifacet group for their insightful comments and feedback on the article. Nilay would like to thank Siddharth Barman, Arkaprava Basu, Brad Beckmann, Dan Gibson, Taedong Kim, Asit K. Mishra, Somayeh Sardashti, Rathijit Sen, and Srikrishna Sridhar for their help.

\section{REFERENCES}

Ahmed H. Abdel-Gawad and Mithuna Thottethodi. 2011. TransCom: Transforming stream communication for load balance and efficiency in networks-on-chip. In Proceedings of the 44th Annual IEEE/ACM International Symposium on Microarchitecture (MICRO-44). ACM, New York, NY, 237247. DOI : http://dx.doi.org/10.1145/2155620.2155648

Dennis Abts, Natalie D. Enright Jerger, John Kim, Dan Gibson, and Mikko H. Lipasti. 2009. Achieving predictable performance through better memory controller placement in many-core CMPs. In Proceedings of the 36th Annual International Symposium on Computer Architecture (ISCA'09). ACM, New York, NY, 451-461. DOI : http://dx.doi.org/10.1145/1555754.1555810

Niket Agarwal, Tushar Krishna, Li-Shiuan Peh, and Niraj K. Jha. 2009. GARNET: A detailed on-chip network model inside a full-system simulator. In Proceedings of the IEEE International Symposium on Performance Analysis of Systems and Software (ISPASS'09). IEEE, Los Alamitos, CA, 33-42.

Manu Awasthi, David W. Nellans, Kshitij Sudan, Rajeev Balasubramonian, and Al Davis. 2010. Handling the problems and opportunities posed by multiple on-chip memory controllers. In Proceedings of the 19th International Conference on Parallel Architectures and Compilation Techniques (PACT'10). ACM, New York, NY, 319-330. DOI : http://dx.doi.org/10.1145/1854273.1854314

Omid Azizi, Aqeel Mahesri, Benjamin C. Lee, Sanjay J. Patel, and Mark Horowitz. 2010. Energy-performance tradeoffs in processor architecture and circuit design: A marginal cost analysis. In Proceedings of the 37th Annual International Symposium on Computer Architecture (ISCA'10). ACM, New York, NY, 26-36. DOI : http://dx.doi.org/10.1145/1815961.1815967

D. H. Bailey, E. Barszcz, J. T. Barton, D. S. Browning, R. L. Carter, L. Dagum, R. A. Fatoohi, P. O. Frederickson, T. A. Lasinski, R. S. Schreiber, H. D. Simon, V. Venkatakrishnan, and S. K. Weeratunga. 1991. The NAS Parallel Benchmarks_-summary and preliminary results. In Proceedings of the 1991 ACM/IEEE Conference on Supercomputing (Supercomputing'91). ACM, New York, NY, 158-165. DOI :http://dx.doi.org/ $10.1145 / 125826.125925$

Yaniv Ben-Itzhak, Israel Cidon, and Avinoam Kolodny. 2012. Optimizing heterogeneous NoC design. In Proceedings of the International Workshop on System Level Interconnect Prediction (SLIP'12). ACM, New York, NY, 32-39. DOI : http://dx.doi.org/10.1145/2347655.2347670

Dimitri Bertsekas and Robert Gallager. 1992. Data Networks. Prentice Hall.

Nathan Binkert, Bradford Beckmann, Gabriel Black, Steven K. Reinhardt, Ali Saidi, Arkaprava Basu, Joel Hestness, Derek R. Hower, Tushar Krishna, Somayeh Sardashti, Rathijit Sen, Korey Sewell, Muhammad Shoaib, Nilay Vaish, Mark D. Hill, and David A. Wood. 2011. The gem5 simulator. ACM SIGARCH Computer Architecture News 39, 2, 1-7. DOI : http://dx.doi.org/10.1145/2024716.2024718

Paul Bogdan. 2015. Mathematical modeling and control of multifractal workloads for data-center-on-a-chip optimization. In Proceedings of the 9th International Symposium on Networks-on-Chip (NOCS'15). ACM, New York, NY, Article No. 21. DOI : http://dx.doi.org/10.1145/2786572.2786592

Paul Bogdan, Miray Kas, Radu Marculescu, and Onur Mutlu. 2010. QuaLe: A quantum-leap inspired model for non-stationary analysis of NoC traffic in chip multi-processors. In Proceedings of the 2010 4th ACM/IEEE International Symposium on Networks-on-Chip (NOCS'10). IEEE, Los Alamitos, CA, 241-248. DOI : http://dx.doi.org/10.1109/NOCS.2010.34

Stephen Boyd and Lieven Vandenberghe. 2004. Convex Optimization. Cambridge University Press, New York, NY.

William Dally and Brian Towles. 2003. Principles and Practices of Interconnection Networks. Morgan Kaufmann, San Francisco, CA. 
J. Feehrer, S. Jairath, P. Loewenstein, R. Sivaramakrishnan, D. Smentek, S. Turullols, and A. Vahidsafa. 2013. The Oracle Sparc T5 16-core processor scales to eight sockets. IEEE Micro 33, 2, 48-57. DOI : http:// dx.doi.org/10.1109/MM.2013.49

Changqing Fu and Kent Wilken. 2002. A faster optimal register allocator. In Proceedings of the 35th Annual ACM / IEEE International Symposium on Microarchitecture (MICRO-35). IEEE, Los Alamitos, CA, 245256. http://dl.acm.org/citation.cfm?id=774861.774888

GAMS Development Corporation. 2015. General Algebraic Modeling System (GAMS) Release 24.4.3. Available at http://www.gams.com.

Dan Gibson. 2012. Private communication.

M. X. Goemans, A. V. Goldberg, S. Plotkin, D. B. Shmoys, É. Tardos, and D. P. Williamson. 1994. Improved approximation algorithms for network design problems. In Proceedings of the 5th Annual ACM-SIAM Symposium on Discrete Algorithms (SODA'94). 223-232. http://dl.acm.org/citation.cfm?id=314464.314497

Boris Grot, Joel Hestness, Stephen W. Keckler, and Onur Mutlu. 2011. Kilo-NOC: A heterogeneous network-on-chip architecture for scalability and service guarantees. In Proceedings of the 38th Annual International Symposium on Computer Architecture (ISCA'11). ACM, New York, NY, 401-412. DOI : http://dx.doi.org/10.1145/2000064.2000112

Akshay Gupte, Shabbir Ahmed, Myun Seok Cheon, and Santanu S. Dey. 2012. Solving Mixed Integer Bilinear Problems Using MIP Formulations. Available at http://www.optimization-online.org/ DB_HTML/2011/07/3087.html.

Gurobi Optimization, Inc. 2015. Gurobi Optimizer Reference Manual. Available at http://www.gurobi.com.

Mitchell Hayenga, Natalie Enright Jerger, and Mikko Lipasti. 2009. SCARAB: A single cycle adaptive routing and bufferless network. In Proceedings of the 42nd Annual IEEE/ACM International Symposium on Microarchitecture (MICRO-42). ACM, New York, NY, 244-254. DOI:http://dx.doi.org/ $10.1145 / 1669112.1669144$

John L. Henning. 2006. SPEC CPU2006 benchmark descriptions. ACM SIGARCH Computer Architecture News 34, 4, 1-17. DOI : http://dx.doi.org/10.1145/1186736.1186737

W.-L. Hung, Y. Xie, N. Vijaykrishnan, C. Addo-Quaye, T. Theocharides, and M. J. Irwin. 2005. Thermal-aware floorplanning using genetic algorithms. In Proceedings of the 2005 International Symposium on Quality of Electronic Design. 634-639. DOI : http://dx.doi.org/10.1109/ISQED.2005.122

IBM Decision Optimization. 2015. IBM ILOG CPLEX Optimizer. Retrieved July 26, 2016, from http:// www.cplex.com.

Hyunjun Jang, Jinchun Kim, Paul Gratz, Ki Hwan Yum, and Eun Jung Kim. 2015. Bandwidth-efficient onchip interconnect designs for GPGPUs. In Proceedings of the 52 nd Annual Design Automation Conference (DAC'15). ACM, New York, NY, Article No. 9. DOI : http://dx.doi.org/10.1145/2744769.2744803

Natalie D. Enright Jerger and Li-Shiuan Peh. 2009. On-Chip Networks. Morgan \& Claypool.

Michel A. Kinsy, Myong Hyon Cho, Tina Wen, Edward Suh, Marten van Dijk, and Srinivas Devadas. 2009. Application-aware deadlock-free oblivious routing. In Proceedings of the 36th Annual International Symposium on Computer Architecture (ISCA'09). ACM, New York, NY, 208-219. DOI : http://dx.doi.org/10.1145/1555754.1555782

Sheng Ma, Natalie Enright Jerger, and Zhiying Wang. 2011. DBAR: An efficient routing algorithm to support multiple concurrent applications in networks-on-chip. In Proceedings of the 38th Annual International Symposium on Computer Architecture (ISCA'11). ACM, New York, NY, $413-424$. DOI : http://dx.doi.org/10.1145/2000064.2000113

Sheng Ma, Natalie Enright Jerger, and Zhiying Wang. 2012. Whole packet forwarding: Efficient design of fully adaptive routing algorithms for networks-on-chip. In Proceedings of the 2012 IEEE 18th International Symposium on High-Performance Computer Architecture (HPCA'12). IEEE, Los Alamitos, CA, 1-12. DOI : http://dx.doi.org/10.1109/HPCA.2012.6169049

T. L. Magnanti and R. T. Wong. 1984. Network design and transportation planning: Models and algorithms. Transportation Science 18, 1-56.

Radu Marculescu and Paul Bogdan. 2009. The chip is the network: Toward a science of network-onchip design. Foundations and Trends in Electronic Design Automation 2, 4, 371-461. http://dx.doi.org/ 10.1561/1000000011

Asit K. Mishra. 2012. Private communication.

Asit K. Mishra, Narayanan Vijaykrishnan, and Chita R. Das. 2011. A case for heterogeneous on-chip interconnects for CMPs. In Proceedings of the 38th Annual International Symposium on Computer Architecture (ISCA'11). ACM, New York, NY, 389-400. DOI : http://dx.doi.org/10.1145/2000064.2000111

Thomas Moscibroda and Onur Mutlu. 2009. A case for bufferless routing in on-chip networks. In Proceedings of the 36th Annual International Symposium on Computer Architecture (ISCA'09). ACM, New York, NY, 196-207. DOI : http://dx.doi.org/10.1145/1555754.1555781 
Tony Nowatzki, Michael Ferris, Karthikeyan Sankaralingam, Cristian Estan, Nilay Vaish, and David Wood. 2013. Optimization and mathematical modeling in computer architecture. Synthesis Lectures on Computer Architecture 8, 4, 1-144. DOI : http://dx.doi.org/10.2200/S00531ED1V01Y201308CAC026

Christos H. Papadimitriou. 1981. On the complexity of integer programming. Journal of the ACM 28, 4 , 765-768. DOI : http://dx.doi.org/10.1145/322276.322287

Alexander Shapiro, D. Dentcheva, and A. Ruszczynski. 2009. Lectures on Stochastic Programming. SIAM.

Allan Snavely and Dean M. Tullsen. 2000. Symbiotic jobscheduling for a simultaneous multithreaded processor. In Proceedings of the 9th International Conference on Architectural Support for Programming Languages and Operating Systems (ASPLOS IX). ACM, New York, NY, 234-244. DOI:http://dx.doi.org/ 10.1145/378993.379244

K. Srinivasan, K. S. Chatha, and G. Konjevod. 2004. Linear programming based techniques for synthesis of network-on-chip architectures. In Proceedings of the 2004 IEEE International Conference on Computer Design: VLSI in Computers and Processors (ICCD'04). 422-429. http://dx.doi.org/ 10.1109/ICCD.2004.1347957

Mohit Tawarmalani and Nikolaos V. Sahinidis. 2005. A polyhedral branch-and-cut approach to global optimization. Mathematical Programming: Series $A$ and $B$ 103, 2, 225-249. DOI:http://dx.doi.org/ 10.1007/s10107-005-0581-8

H.-M. Tong, Y.-S. Lai, and C. P. Wong. 2013. Advanced Flip Chip Packaging. Springer.

Zheng Wang and Michael F. P. O’Boyle. 2009. Mapping parallelism to multi-cores: A machine learning based approach. In Proceedings of the 14th ACM SIGPLAN Symposium on Principles and Practice of Parallel Programming (PPoPP'09). ACM, New York, NY, 75-84. DOI : http://dx.doi.org/10.1145/1504176.1504189

Thomas Canhao Xu, Pasi Liljeberg, and Hannu Tenhunen. 2011. Optimal memory controller placement for chip multiprocessor. In Proceedings of the 7th IEEE / ACM / IFIP International Conference on Hardware / Software Codesign and System Synthesis (CODES+ISSS'11). ACM, New York, NY, 217-226. DOI : http://dx.doi.org/10.1145/2039370.2039405

Wenbiao Zhou, Yan Zhang, and Zhigang Mao. 2006. Pareto based multi-objective mapping IP cores onto NoC architectures. In Proceedings of the 2006 IEEE Asia Pacific Conference on Circuits and Systems. 331-334. DOI : http://dx.doi.org/10.1109/APCCAS.2006.342418

Received November 2015; revised March 2016; accepted May 2016 